\title{
LAS ESPECIES DE Arctodictis MERCERAT, 1891 (METATHERIA, BORHYAENIDAE), GRANDES CARNÍVOROS DEL MIOCENO DE AMÉRICA DEL SUR
}

\author{
Analía FORASIEPI ${ }^{1}$, Francisco J. GOIN ${ }^{2}$ y Adán \\ A. TAUBER ${ }^{3}$ \\ ${ }^{1}$ Sección Paleontología de Vertebrados, Museo Argentino de Ciencias Natu- \\ rales "Bernardino Rivadavia". Av. Angel Gallardo 470, 1405 Buenos Aires- \\ Argentina. \\ ${ }^{2}$ Departamento Paleontología de Vertebrados, Museo de La Plata, Paseo del Bos- \\ que s/n, 1900 La Plata- Argentina. fgoin@museo.fcnym.unlp.edu.ar \\ ${ }^{3}$ Centro Regional de Investigaciones Científicas y Transferencia Tecnológi- \\ ca La Rioja (CRILAR). Mendoza y Entre Ríos, 5301, Anillaco, La Rioja- Ar- \\ gentina y Museo de Ciencias Naturales, Universidad Nacional de La Rioja, \\ Av. Laprida y V. Bustos, 5300, La Rioja- Argentina.
}

Forasiepi, A., Goin, F. J. y Tauber, A. A. 2004. Las especies de Arctodictis Mercerat, 1891 (Metatheria, Borhyaenidae), grandes carnívoros del Mioceno de América del Sur. [The species of Arctodictis Mercerat, 1891 (Metatheria, Borhyaenidae), large carnivorous from the Miocene of South America.] Revista Española de Paleontología, 19 (1), 1-22. ISSN 0213-6937.

\begin{abstract}
During most of the Cenozoic, South American large carnivorous vertebrates were represented by phororhacid birds, crocodiles, giant snakes and especially, metatherian mammals such as the Borhyaenidae. Between them, Arctodictis munizi Mercerat, 1891 represents the largest borhyaenid species known up to now. A new specimen of A. munizi collected in the Santa Cruz Formation (Middle Miocene) at Cañadón Las Totoras (Santa Cruz Province, Argentina), allowed us to describe its cranial anatomy, to discuss the phylogenetic relationships of the genus Arctodictis, and to review the status of several problematic specimens. A study of the bearing sediments suggests environmental conditions characterized by a strong seasonality, open environments, frequent small streams, and grasslands. From the morphological and phylogenetic analysis we concluded that: (1) Arctodictis is a natural group supported by the following derived features: fused or strongly ankylosed dentaries at the symphysis; $\mathrm{P} / \mathrm{p} 1$ transversely oriented, m1-3 with a descendent labial lobe of the crown on the posterior root, opening of the infraorbitary foramen above M1, expanded palatines behind the last molar, and globe-shaped roots in the premolars. (2) The genus includes at least two species: A. munizi and A. sinclairi. Specimen UCMP 39250, referred by Marshall to cf. Arctodictis sp., would represent a big 'prothylacinin-like' sparassodont. (3) There is a wide variability in size and robustness among different individuals.
\end{abstract}

Key words: Metatheria, Sparassodonta, Borhyaenidae, Arctodictis, Miocene, South America, Patagonia.

\section{RESUMEN}

Durante la mayor parte del Cenozoico, los grandes vertebrados carnívoros de América del Sur estuvieron representados por aves fororacoideas, cocodrilos, serpientes gigantes y mamíferos metaterios como los Borhyaenidae. Entre estos últimos, Arctodictis munizi Mercerat, 1891 representa al boriénido de mayor tamaño hasta ahora conocido. Un nuevo espécimen de A. munizi recolectado en la Formación Santa Cruz (Mioceno Medio) en Cañadón Las Totoras (Provincia de Santa Cruz, Argentina), nos ha permitido describir su anatomía craneal, discutir las relaciones filogenéticas del género Arctodictis y revisar la situación taxonómica de varios especímenes problemáticos. Un estudio de los sedimentos relacionados sugiere condiciones ambientales caracterizadas por una estacionalidad fuerte, ambientes abiertos, frecuentes arroyos pequeños y pastizales. A partir del análisis morfológico y filogenético hemos concluido que: (1) Arctodictis es un grupo natural soportado por los siguientes caracteres derivados: dentarios fusionados o fuertemente anquilosados en la sínfisis; P/p 1 orientado 
transversalmente, m1-3 con un lóbulo labial de la corona sobre la raíz posterior, foramen infraorbitario ubicado sobre el M1, palatinos expandidos detrás del último molar y raíces de los premolares de forma globosa. (2) El género incluye por lo menos dos especies: A. munizi y A. sinclairi. El espécimen UCMP 39250 referido por Marshall a cf. Arctodictis sp. podría representar un gran esparasodonte próximo a los protilacíninos. (3) Existe una amplia variabilidad en tamaño y robustez entre diferentes individuos.

\section{Palabras clave: Metatheria, Sparassodonta, Borhyaenidae, Arctodictis, Mioceno, América del Sur, Pata- gonia.}

\section{INTRODUCCIÓN}

Durante el aislamiento biogeográfico ocurrido en América del Sur en gran parte del Terciario, los metaterios se diversificaron ocupando zonas adaptativas sumamente diversas, y que en otras partes del mundo eran ocupadas por los mamíferos euterios (Marshall, 1977a). El papel ecológico correspondiente a los grandes carnívoros, tanto carniceros como carroñeros, fue ocupado parcialmente por los esparasodontes (Metatheria, Sparassodonta; véase Simpson, 1980; Marshall y Cifelli, 1990).

Los primeros restos fósiles de estos mamíferos fueron dados a conocer por Florentino Ameghino (1887) sobre la base de materiales recolectados por su hermano Carlos en la provincia de Santa Cruz (Argentina). Posteriormente y a lo largo de todo el siglo XX fueron numerosos los hallazgos realizados no sólo en la Patagonia (Argentina) sino también en gran parte de América del Sur (véase Marshall, 1976a, 1977b, 1978, 1979, 1981; Petter y Hoffstetter, 1983; Muizon, 1994, 1998), los que han incrementado notablemente el conocimiento tanto anatómico como sistemático del grupo.

La sistemática supragenérica de estos metaterios es compleja. Entre las más modernas revisiones del grupo se destaca aquella de Marshall (1978). Como resultado de su estudio, Marshall (1978) incluyó en la superfamilia Borhyaenoidea a las familias Thylacosmilidae y Borhyaenidae, esta última abarcando a las entonces consideradas subfamilias Hathliacyninae, Proborhyaeninae, Prothylacyninae y Borhyaeninae. Posteriormente Marshall et al. (1990) reconocieron el rango ordinal Sparassodonta (sistemática que se sigue en este trabajo), e incluyeron esta vez a las familias Thylacosmilidae, Proborhyaenidae, Hathliacynidae y Borhyaenidae (Prothylacyninae + Borhyaeninae), al tiempo que adicionaron las familias Hondadelphyidae y Stagodontidae (aunque posteriormente esta última fue excluida del grupo; Marshall y Kielan-Jaworowska, 1992). Más recientemente, Muizon (1994) creó la familia Mayulestidae, con un sólo género monotípico, Mayulestes Muizon, 1994, el cual representa a su vez el más primitivo Sparassodonta (sus Borhyaenoidea; Muizon, 1994, 1998, 1999) hasta ahora conocido. A pesar de las recientes revisiones de las que han sido objeto estos metaterios, tanto la composición taxonómica como las relaciones filogenéticas están lejos de haberse resuelto.
Por su parte, el género Arctodictis (Borhyaenidae, Borhyaeninae) incluye las formas más grandes de boriénidos neógenos (Marshall, 1978). Fue dado a conocer por Mercerat (1891) quien consideró entonces dos especies: A. munizi Mercerat, 1891 y A. australis Mercerat, 1891, aunque esta última fue posteriormente reasignada por Ameghino (1894; véase también Ameghino, 1891) al género Borhyaena Ameghino, 1887. Más recientemente, Marshall (1978) consideró como especies válidas del género a A. munizi (Edad Santacrucense, Mioceno Medio) y A. sinclairi Marshall, 1978 (Edad Colhuehuapense, Mioceno Temprano), ambas basadas en restos patagónicos, al tiempo que refirió a cf. Arctodictis sp. un ejemplar de gran tamaño (UCMP 39250) procedente de la localidad de La Venta (Mioceno Medio; Edad Laventense), Colombia (pero véase más abajo). Es preciso mencionar que en las colecciones del Museo de La Plata se alojan restos muy completos de un boriénido colhuehuapense (MLP 85-VII3-1) cuya morfología mandibular y dentaria sugieren fuertemente su asignación a la especie $A$. sinclairi. Finalmente, un ejemplar referido a cf. Arctodictis fue mencionado para el Mioceno Medio de la provincia del Neuquén (Formación Collón Curá; Edad Friasense) por Pardiñas (1991). De confirmarse su pertenencia tanto a este género como a estos niveles, se trataría del resto más moderno hasta ahora conocido asignable al género. Sin embargo, el ejemplar no fue encontrado por nosotros en las colecciones del Museo de La Plata al momento de la realización de esta revisión.

Si bien la mayor parte de los ejemplares asignados al género Arctodictis corresponden a materiales fragmentarios (véase la Tabla 1), recientemente Tauber (1997a, b) dió a conocer un nuevo ejemplar de A. munizi procedente de rocas de edad Santacrucense, aflorantes en la localidad Cañadón Las Totoras (Estancia Angelina, provincia de Santa Cruz, Argentina; Fig. 1). El mismo consiste en un cráneo y mandíbula asociados a unos pocos elementos del esqueleto postcraneano (Figs. 2-6), por lo que constituye el ejemplar más completo hasta ahora conocido asignable a esta especie. En este trabajo se describen dichos materiales y se discute la sistemática del género sobre la base de la información así obtenida.

Cabe también agregar que la mayoría de los especímenes disponibles de Arctodictis munizi pertenecen a colecciones antiguas como las de Berry, Ameghino y otros, 


\begin{tabular}{|c|c|c|c|c|c|}
\hline $\mathrm{N}^{\mathrm{o}}$ de colección & Especie & Materiales & Localidad y estratigrafía & Colector & Observaciones \\
\hline MLP 11-65 & Arctodictis munizi & Cráneo y mandíbula & Santacrucense & Berry, 1864 & Ingresado como Borhyaena fera \\
\hline MLP 11-81 & Arctodictis munizi & m4 izquierdo & Santacrucense & & Ingresado como Borhyaena fera \\
\hline MLP 11-85 & Arctodictis munizi & Fragmento mandibular & $\begin{array}{l}\text { Monte León } \\
\text { Santacrucense }\end{array}$ & Viejas colecciones & Holotipo \\
\hline MLP 11-89 & Arctodictis munizi & $\mathrm{m} 2$ derecho & Santacrucense & & $\begin{array}{c}\text { Ingresado como } \\
\text { Prothylacynus patagonicus }\end{array}$ \\
\hline $\begin{array}{l}\text { MACN A } \\
5915-5017\end{array}$ & Arctodictis munizi & $\begin{array}{l}\text { Maxilar y dos } \\
\text { mandíbulas }\end{array}$ & $\begin{array}{l}\text { Corriguen Aike } \\
\text { Santacrucense }\end{array}$ & Carlos Ameghino & $\begin{array}{l}\text { Ingresado como } \\
\text { Borhyaena fera }\end{array}$ \\
\hline $\begin{array}{l}\text { MACN A } \\
5918-5921\end{array}$ & Arctodictis munizi & Cráneo, dentario & $\begin{array}{l}\text { Corriguen Aike } \\
\text { Santacrucense }\end{array}$ & Carlos Ameghino & $\begin{array}{l}\text { Ingresado como } \\
\text { Borhyaena fera }\end{array}$ \\
\hline $\begin{array}{l}\text { CORD-PZ } \\
1210-1 / 5\end{array}$ & Arctodictis munizi & $\begin{array}{c}\text { Cráneo, mandíbula } \\
\text { y fragmentos } \\
\text { postcraneanos }\end{array}$ & $\begin{array}{l}\text { Cañadón Las Totoras } \\
\text { Fm Santa Cruz }\end{array}$ & Tauber, 1988 & \\
\hline FMHP 13526 & Arctodictis sinclairi & Fragmento mandibular & Colhuehuapense & & Holotipo \\
\hline $\begin{array}{l}\text { MLP 85- } \\
\text { VII-3-1 }\end{array}$ & $\begin{array}{l}\text { cf. Arctodictis } \\
\text { sinclairi }\end{array}$ & $\begin{array}{c}\text { Esqueleto } \\
\text { casi completo }\end{array}$ & Colhuehuapense & Juan Quiroga & \\
\hline UCMP 39250 & $\begin{array}{l}\text { cf. Dukecynus } \\
\text { magnus }\end{array}$ & $\begin{array}{c}\text { Fragmentos craneanos, } \\
\text { mandibulares y } \\
\text { postcraneanos }\end{array}$ & $\begin{array}{c}\text { La Venta, } \\
\text { Colombia. Laventense }\end{array}$ & & \\
\hline MACN A 5922 & Borhyaena tuberata & Fragmento craneano & Santacrucense & & Holotipo de Borhyaena zittelli \\
\hline MACN A 6238 & $\begin{array}{l}\text { Borhyaena } \\
\text { tuberata }\end{array}$ & $\begin{array}{c}\text { Fragmento craneano, } \\
\text { mandíbula y parte } \\
\text { del postcráneo }\end{array}$ & Santacrucense & & \\
\hline MACN A 9342-3 & $\begin{array}{l}\text { Borhyaena } \\
\text { tuberata }\end{array}$ & $\begin{array}{l}\text { Fragmento de dos } \\
\text { maxilares y de dos } \\
\text { mandíbulas }\end{array}$ & Santacrucense & & \\
\hline MACN A 9344 & Borhyaena tuberata & Cráneo & Santacrucense & & \\
\hline MACN A s $/ n^{\circ}$ & Borhyaena tuberata & Fragmento de maxilar & Santacrucense & & \\
\hline MACN A s $/ n^{\circ}$ & Borhyaena tuberata & $\begin{array}{c}\text { Ambas } \\
\text { hemimandíbulas }\end{array}$ & Santacrucense & & \\
\hline MACN A 706 & $\begin{array}{l}\text { Prothylacynus } \\
\text { patagonicus }\end{array}$ & $\begin{array}{l}\text { Frag. de cráneo, } \\
\text { mandíbula y parte } \\
\text { del postcráneo }\end{array}$ & Santacrucense & Carlos Ameghino & Holotipo \\
\hline $\begin{array}{l}\text { MACN A } \\
5931-5937\end{array}$ & $\begin{array}{l}\text { Prothylacynus } \\
\text { patagonicus }\end{array}$ & $\begin{array}{l}\text { Cráneo, mandíbula } \\
\text { y } 2 \text { ulnas }\end{array}$ & Santacrucense & Carlos Ameghino & $\begin{array}{l}\text { Holotipo de Napodonictis } \\
\text { thylacynoides }\end{array}$ \\
\hline UCMP 38061 & Lycopsis longirostrus & $\begin{array}{c}\text { Esqueleto casi } \\
\text { completo y articulado }\end{array}$ & $\begin{array}{l}\text { La Venta, Colombia, } \\
\text { Laventense }\end{array}$ & & Holotipo \\
\hline
\end{tabular}

Tabla 1. Lista de los especímenes considerados en este trabajo. List of the specimens considered in this paper. 

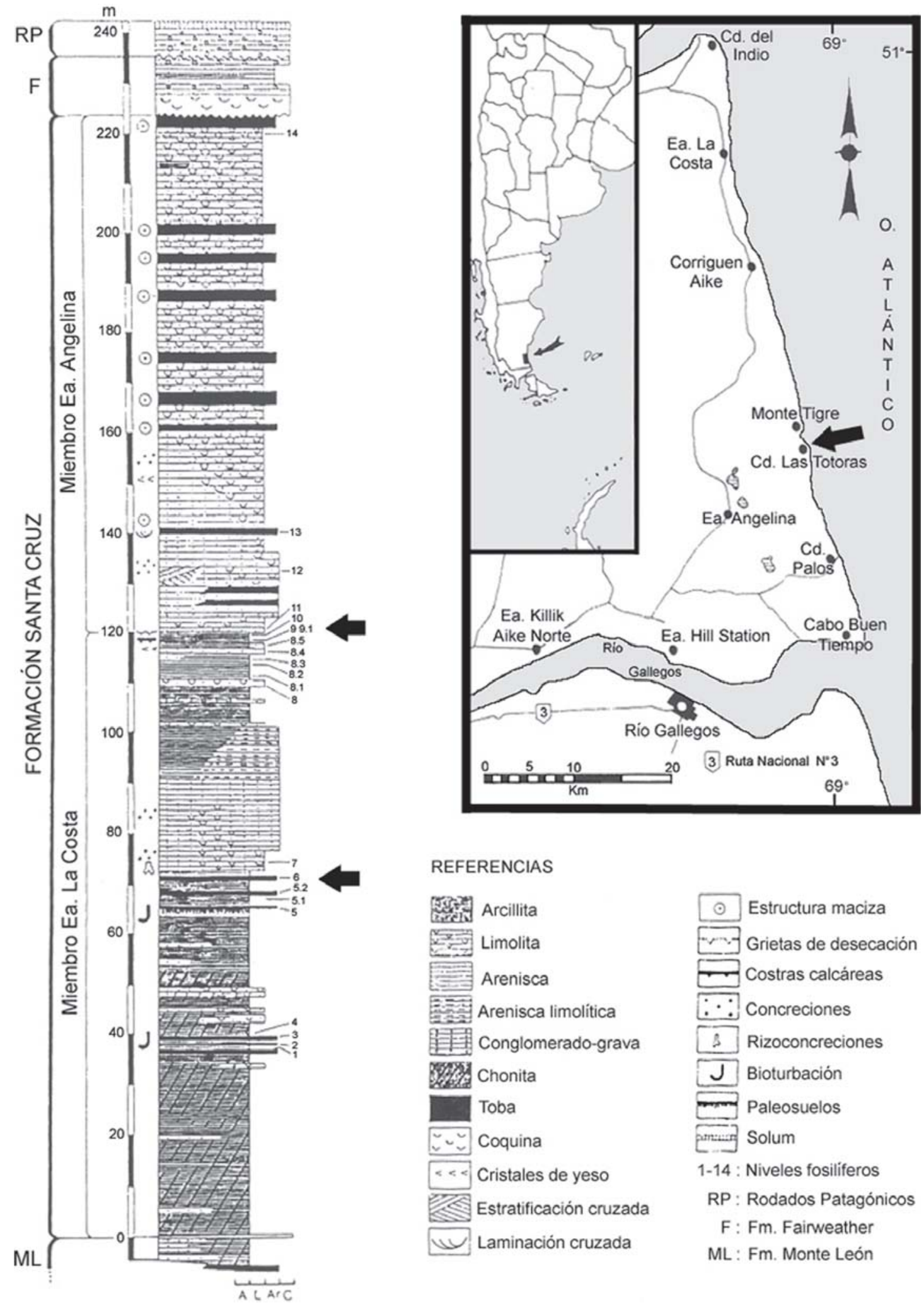

\section{REFERENCIAS}
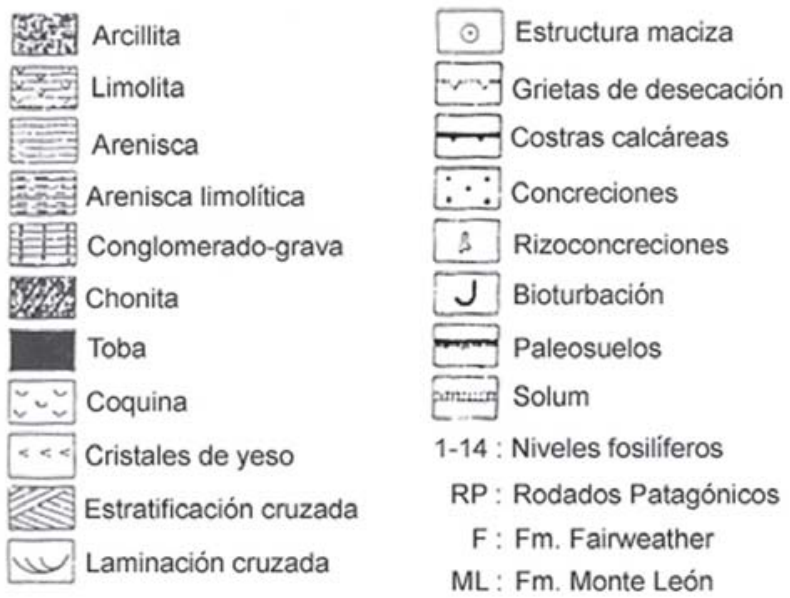

Figura 1. Mapa de ubicación de las localidades con afloramientos santacrucenses citadas en el texto (provincia de Santa Cruz, Argentina); la flecha indica la localidad de procedencia del ejemplar de Arctodictis munizi Mercerat, 1891 CORD-PZ 1210-1/2. Columna estratigráfica de la Formación Santa Cruz (tomada de Tauber, 1999), las flechas indican los niveles de procedencia de los ejemplares de $A$. munizi mencionados en este trabajo.

Location map of the santacrucian outcrops mentioned in the text (Santa Cruz Province, Argentina); the arrow shows the locality of the specimen Arctodictis munizi Mercerat, 1891 CORD-PZ 1210-1/2. Stratigraphic column of the Santa Cruz Formation (taken from Tauber, 1999), the arrows show the level where the specimens of A. munizi mentioned in this paper come from. 
depositadas el Museo de La Plata y Museo Argentino de Ciencias Naturales "Bernardino Rivadavia". Todos ellos tienen como único dato de procedencia el "Santacrucense" (sensu lato), por lo que es casi inexistente una información estratigráfica más precisa, más allá de su exhumación en niveles de la Formación Santa Cruz (Mioceno Medio). El nuevo material de A. munizi (CORD-PZ 1210$1 / 5$ ), adquiere entonces una significación especial, al tratarse de uno de los pocos boriénidos hasta ahora recolectados con datos estratigráficos precisos. Esto permite su análisis en el marco de una asociación específica de los fósiles de la Formación Santa Cruz.

\begin{abstract}
Abreviaturas. CORD-PZ, Museo de Paleontología, Facultad de Ciencias Exactas, Físicas y Naturales de la Universidad Nacional de Córdoba- Argentina; FMNH, Field Museum of Natural History, Chicago- EEUU; MACN-A, Colección Ameghino, Sección Paleontología Vertebrados, Museo Argentino de Ciencias Naturales "Bernardino Rivadavia", Buenos Aires- Argentina; MLP, Departamento Paleontología de Vertebrados, Museo de La Plata- Argentina; UCMP, University of California Museum of Paleontology, Berkeley- EEUU; I/i, incisivo superior/inferior; $\mathrm{C} / \mathrm{c}$, canino superior/inferior; $\mathrm{P} / \mathrm{p}$, premolar superior/inferior; $\mathrm{M} / \mathrm{m}$ molar superior/inferior.
\end{abstract}

\section{CONTEXTO GEOLÓGICO, BIOESTRATIGRÁFICO Y PALEOAMBIENTAL}

Tanto por su cantidad como por su calidad y diversidad de vertebrados fósiles, la Formación Santa Cruz (Mioceno Medio) es una de las unidades litoestratigráficas cenozoicas más ricas de América del Sur (Marshall, 1976b, 1985; Marshall et al. 1983, 1986; Scillato Yané, 1986; Tauber, 1997a, b). La Formación Santa Cruz está compuesta por dos miembros (Fig. 1): el inferior es el Miembro Estancia La Costa (de $120 \mathrm{~m}$ de potencia), que incluye 19 niveles fosilíferos; el superior, Miembro Estancia Angelina (103 m de espesor) incluye cuatro niveles fosilíferos (véase Tauber, 1997a, b, 1999). A pesar de la abundancia de vertebrados fósiles, los restos de metaterios carnívoros son relativamente escasos. Una muestra recientemente exhumada de esta formación (Tauber, 1999), consistente en unos 500 especímenes de vertebrados procedentes de siete localidades y 23 niveles fosilíferos diferentes, incluyó sólo dos ejemplares de boriénidos, de los cuales uno de ellos es el que se estudia en este trabajo.

\section{Marco geológico}

El ejemplar CORD-PZ 1210-1/5 procede de la zona intermareal de un sitio costero próximo al Cañadón Las

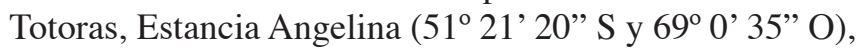
en el sudeste de la provincia de Santa Cruz, (Fig. 1). La unidad litoestratigráfica aflorante es la parte alta (los 10 m superiores) del Miembro Estancia La Costa. Esta unidad está compuesta esencialmente por arcillitas montmorilloníticas de estructura maciza y contactos graduales. Existe una gran concentración de paleocanales y depósitos de origen lacustre. Algunos paleocauces están rellenos con areniscas bien seleccionadas y con estratificación entrecruzada en artesa muy bien definidas (facies St). Otro tipo de cuerpo sedimentario está compuesto por depósitos de tamaño más reducido, rellenos de areniscas verdes de granulometría muy fina y texturalmente muy inmaduras, que gradan a limolitas de estructura maciza (facies Fsc). Estos cuerpos son portadores de restos de vertebrados, algunos de los cuales están articulados, evidenciando un ambiente de sedimentación de baja energía y probable origen lacustre. El rumbo de los estratos es de $10^{\circ}$, con una intensidad de buzamiento de $1^{\circ} 40^{\prime}$ en dirección a los $100^{\circ}$. Análisis previos en este sector han determinado la presencia de ocho niveles fosilíferos (Tauber, 1996). El ejemplar CORD-PZ 1210-1/5 procede del nivel fosilífero 9 (Tauber, 1997a, b, 1999. Fig. 1).

Por el contrario, no se conoce con precisión el nivel fosilífero de procedencia de los especímenes recolectados por Carlos Ameghino y John B. Hatcher en Corriguen Aike (MACN A 5915-5017 y MACN A 5918-5921). Es también preciso destacar que este topónimo tuvo algunas variaciones en el nombre, llamándoselo alternativamente Corrigenkaik, Corrigen Kaik, Corriken Aike o Corriguen Aike (Hatcher, 1903; Scott, 1905; Ameghino, 1906; Sinclair, 1906, 1909; Marshall, 1976b etc.) o Puesto de la Estancia La Costa (Tauber, 1991). Existen algunos indicios que permiten inferir que dichas colecciones procederían de la zona intermareal frente al cañadón de Corrigen Aike ( $51^{\circ} 12^{\prime} 8,2^{\prime \prime} \mathrm{S}$ y $\left.69^{\circ} 03^{\prime} 35,6^{\prime \prime} \mathrm{O}\right)$, por ser éstos los niveles más accesibles y ricos en restos de vertebrados fósiles y de excelente estado de conservación. En dicha zona, afloran niveles de arcillita verde a verde grisáceo de estructura maciza, sobre la que se depositó una toba cinerítica verde grisácea maciza que constituye el nivel fosilífero 6 (NF6) (Tauber, 1997a, b). Se ha observado además una intercalación de arenisca fina, muy inmadura texturalmente y de estructura maciza, que podría coresponder con un cuerpo de origen fluvial de baja energía o lacustre.

\section{Tafonomía}

El ejemplar CORD-PZ 1210-1/5 fue exhumado a unos $3 \mathrm{~m}$ de distancia de un pequeño depósito fluvial; el cráneo estaba articulado y ocluido con la mandíbula junto a varios restos postcraneanos asociados, por lo que se considera que el material era autóctono. El nivel fosilífero de este ejemplar (NF9) posee rasgos litológicos y tafonómicos comunes con los niveles fosilíferos $8,8.1,8.2,8.3$, $8.4,8.5$, y 10 , aflorantes en la zona intermareal entre Monte Tigre y el cañadón Las Totoras. Esto ha permitido 
definir una tafofacies cuya litología está compuesta por arcillitas, limolitas y areniscas finas de colores verde grisáceos muy claros, con abundantes concreciones calcáreas (sensu lato), costras, rellenos de grietas de desecación de la misma composición y con deformaciones por carga litostática, $\left(\sigma_{1}\right.$ vertical) (Tauber, 1994). Se destaca el alto porcentaje de huesos incluidos en concreciones (sensu lato) o asociados a costras calcáreas (en el NF 10). Varios fósiles muestran signos de disolución en los huesos, a tal punto que es frecuente la conservación de toda la serie dentaria en su posición, habiéndose perdido total o parcialmente los elementos craneanos que los alojaban (maxilares, premaxilares y dentarios). Esto resulta particularmente frecuente en los especímenes de Protypotherium australe (Notoungulata, Interatheriidae) y Toxodontidae (Notoungulata). En esta tafofacies son más frecuentes los hallazgos de cráneos aislados y de pequeñas astillas óseas (diámetro mayor a $2 \mathrm{~mm}$ ), mientras que resultan escasos los restos de especies de talla pequeña.

La litología que caracteriza la tafofacies del nivel fosilífero 6 (de donde procederían los ejemplares MACN A 5915-5917 y MACN A 5918-5921) incluye chonitas o tobas finas de color blanco grisáceo con estructura maciza y con distintos grados de alteración. Los elementos óseos se encuentran más completos, frecuentemente articulados y con un bajo grado de alteración meteórica; además no hay evidencias de deformaciones por carga litostática ni de disolución. Entre los restos articulados se destaca un espécimen de Interatherium sp. (Notoungulata, Interatheriidae) con la caja torácica articulada y conservando a su vez su volumen real, lo que pone en evidencia una alta tasa de sedimentación.

\section{Bioestratigrafía}

El ejemplar de Arctodictis munizi aquí descrito (CORDPZ 1210-1/5) procede del nivel fosilífero 9, asignable a la "Biozona de Intervalo de Protypotherium australe", coincidente con la "Biozona de Asociación de Protypotherium australe-Proeutatus oenophorus" (Tauber, 1994, 1996, 1997a). La asociación fosilífera es la siguiente: Artodictis munizi (Metatheria, Borhyaenidae), Prothylacynus patagonicus (Metatheria, Borhyaenidae), Stegotherium tessellatum (Xenarthra, Dasypodidae), Proeutatus oenophorus (Xenarthra, Dasypodidae), Prozaedyus exilis (Xenarthra, Dasypodidae), Cochlops debilis (Xenarthra, Peltephilidae), Eucinepeltus petestatus, Eucinepeltus complicatus (Xenarthra, Glyptodontidae), Hapalops gracilidens, Hapalops indifferens, Hapalops sp. (Xenarthra, Megatheriidae), Pelecyodon cristatus, Pelecyodon sp. (Xenarthra, Megatheriidae), Pseudhapalops rutimeyeri (Xenarthra, Megatheriidae), Megalonychotherium atavus (Xenarthra, Megalonychidae), Eucholoeops sp. (Xenarthra, Megalonychidae), Neoreomys australis (Rodentia, Dasyproctidae), Eocardia montana (Rodentia, Eocardiidae), Eocardiidae gen. et sp. indet. (Rodentia), Prolagostomus obliquidens (Rodentia, Chinchillidae), "Proterotherium" intermedium (Litopterna, Protherotheriidae), Licaphrium floweri (Litopterna, Protherotheriidae), Thoatherium minusculum (Litopterna, Protherotheriidae), Nesodon imbricatus, Nesodon sp. (Notoungulata, Toxodontidae), Adinotherium ovinum, Adinotherium robustum (Notoungulata, Toxodontidae), Interatherium sp. (Notoungulata, Interatheriidae), Protypotherium praerutilum, Protypotherium australe (Notoungulata, Interatheriidae), Hegetotherium mirabile (Notoungulata, Hegetotheriidae), Astrapotherium nanum?, Astrapotherium cf. A. magnum y Astrapotherium sp. (Astrapotheria, Astrapotheriidae).

El nivel fosilífero 6 (NF6), uno de los estratos más ricos en restos de vertebrados fósiles, es parte de la "Biozona de Protypotherium attenuatum" (Tauber, 1997a). La asociación fosilífera es la siguiente: Psilopterus australis (Aviale, Psilopteridae), Phororhacos longissimus (Aviale, Phororhacidae), Stenotatus patagonicus (Xenarthra, Dasypodidae), Proeutatus cf. P. deleo (Xenarthra, Dasypodidae), Prozaedyus proximus (Xenarthra, Dasypodidae), Propalaehoplophorinae gen. et sp. indet. (Xenarthra, Glyptodontidae), Eucholoeops fronto (Xenarthra, Megalonychidae), Homunculus patagonicus (Primates, Atelidae), Acarechimys minutus (Rodentia, Echimyidae), Neoreomys australis (Rodentia, Dasyproctidae), Eocardia perforata (Rodentia, Eocardiidae), Perimys cf. P. erutus (Rodentia, Neoepiblemidae), Licaphrium floweri (Litopterna, Protherotheriidae), Thoatherium cf. T. minusculum (Litopterna, Protherotheriidae), Adinotherium sp. (Notoungulata, Toxodontidae), Interatherium cf. I. robustum (Notoungulata, Interatheriidae), Protypotherium cf. $P$. attenuatum (Notoungulata, Interatheriidae), Hegetotherium mirabile (Notoungulata, Hegetotheriidae), Astrapotherium magnum (Astrapotheria, Astrapotheriidae).

\section{Paleoclima y paleoambiente}

En trabajos previos se analizaron las probables condiciones climáticas y ambientales imperantes durante la sedimentación de la Formación Santa Cruz (véase Tauber, 1994, 1996, 1997b, 1999). Las conclusiones de dichos análisis se resumen en dos hipótesis: (1) Durante la sedimentación del Miembro Estancia La Costa se habría producido un deterioro climático, especialmente en el grado de humedad, variando desde condiciones estables, cálidas y húmedas a condiciones más secas y con estaciones bien diferenciadas. (2) Durante este mismo lapso se habrían producido cambios que van desde ambientes con predominio de vegetación arbórea o arbustiva a ambientes más abiertos con predominio de vegetación herbácea (Tauber, 1997b).

\section{Paleotopografía}

El sitio de donde proceden los restos de Arctodictis munizi (CORD-PZ 1210-1/5) correspondía a un área baja desde el punto de vista paleotopográfico (Tauber, 1994). En efecto: (1) la mayor cantidad y el mayor desarrollo de 
paleocauces y depósitos lacustres fueron observados en la zona comprendida entre Monte Tigre y Cañadón Las Totoras, en los diferentes niveles estratigráficos que allí afloran; (2) se observa un mayor espesor de las limolitas y tobas de los últimos $66 \mathrm{~m}$ del Miembro Estancia Angelina (Tauber, 1994, tramo F) con respecto a la zona norte (Corrigen Aike, Estancia La Costa), donde los estratos están condensados; (3) se aprecia el desarrollo de estructuras tales como grietas de desecación y costras calcáreas y un paso gradual entre los miembros de la Formación Santa Cruz, y (4) el único paleocanal bien encauzado se localiza en la parte alta del Miembro Estancia Angelina. Estas evidencias indican que dichas localidades correspondieron con sitios paleotopográficos bajos, por lo menos durante la sedimentación de la sección superior del Miembro Estancia La Costa y del Miembro Estancia Angelina.

La existencia de este bajo paleotopográfico es coincidente con la posición del bajo estructural de Monte Tigre, de origen Jurásico (Chelotti, 1992). Esto habría formado una zona de mayor subsidencia en el sector, estableciéndose allí un localizado control tectónico sinsedimentario durante el desarrollo de la Formación Santa Cruz, o al menos de su miembro superior (Tauber, 1994). Por el contrario, al norte del Puesto de la Estancia La Costa, el registro estratigráfico es más discontinuo, los espesores son más reducidos (especialmente en los depósitos epiclásticos), existe una mejor definición de la discontinuidad separando los miembros de la Formación Santa Cruz y una menor frecuencia de los paleocauces. Estas evidencias indicarían que se trata de lugares paleotopográficos algo más altos durante la sedimentación del miembro superior de esta formación, coincidiendo esta vez con la posición del alto estructural de Cañadón Salto, señalado por Chelotti (1992).

En resumen, el ejemplar CORD-PZ 1210-1/5 de Arctodictis munizi aquí descrito procede de la zona intermareal de un sitio costero próximo al Cañadón Las Totoras, Estancia Angelina ( $51^{\circ} 21^{\prime} 20^{\prime \prime} \mathrm{S}$ y $69^{\circ} 0$ ' $35^{\prime \prime} \mathrm{O}$ ), en el sudeste de la provincia de Santa Cruz, de niveles correspondientes a la parte alta del Miembro Estancia La Costa de la Formación Santa Cruz (Mioceno Medio), de la "Biozona de Intervalo de Protypotherium australe" coincidente con la "Biozona de Asociación de Protypotherium australe-Proeutatus oenophorus" (Tauber, 1996, 1997a). El ejemplar es autóctono; procede de un nivel que se habría depositado en un sitio paleotopográfico bajo, probablemente en una llanura de inundación de ríos meandriformes. Las condiciones ambientales habrían sido más secas con respecto a los niveles inferiores y con una marcada estacionalidad definida por la frecuencia de lluvias. El ambiente en general habría sido abierto, con predominio de vegetación herbácea, aunque tratándose de un área topográficamente baja y con paleocauces frecuentes, no debe descartarse la presencia de vegetación en galería siguiendo los cursos de agua.

\section{ANÁLISIS PALEONTOLÓGICO}

\section{Sistemática}

SUBCLASE METATHERIA Huxley, 1880

ORDEN SPARASSODONTA Ameghino, 1894

Familia Borhyaenidae Ameghino, 1894

Subfamilia Borhyaeninae Ameghino, 1894

Género Arctodictis Mercerat, 1891

Nueva diagnosis. Boriénidos de mediano a gran tamaño, caracterizados por la siguiente combinación de caracteres: borde posterior del canal infraorbitario ubicado a la altura del $\mathrm{m} 1$, paladar prolongado posteriormente por detrás del último molar, sínfisis mandibular fuertemente anquilosada a fusionada, $\mathrm{P} / \mathrm{p} 1$ implantado transversalmente en la serie dentaria, desarrollo en los m1-3 de un marcado lóbulo descendente de la corona sobre la raíz posterior, y raíces marcadamente globosas en los premolares.

New diagnosis: Borhyaenids of medium to large size that are characterized by the following character association: opening of the infraorbitary foramen above $\mathrm{ml}$, palate expanded behind the last molar, fused or strongly ankylosed dentaries at the symphysis, P/p 1 transversely oriented, m1-3 with a descendent labial lobe of the crown on the posterior root, and globe-shaped roots in the premolars.

\section{Arctodictis munizi Mercerat, 1891}

Figs. 2-7

Holotipo: MLP 11-85 (Fig. 7), un fragmento mandibular izquierdo con la raíz del canino y los p1-m4 (este último roto en su mitad posterior) y el sector sinfisario de la rama mandibular derecha, con las raíces del canino y los p1-2 y el p3 casi completo.

Hipodigma: el holotipo y CORD-PZ 1210-1/5, un cráneo casi completo (1210-1) con su correspondiente hemimandíbula izquierda y el sector sinfisario de la derecha (1210-2; Figs. 2 y 3), un extremo proximal de húmero derecho (1210-3; Fig. 4a), un fragmento distal de la tibia derecha (1210-4; Fig. 4b), dos vértebras (1210-4/5; Fig. 6) y varios fragmentos costales (s/n); MLP 11-65, un cráneo reconstruido e incompleto con la mandíbula asociada; MLP 11-81, un m4 izquierdo; MLP 11-89, un m2 derecho; MACN A 5915-5917 (elementos correspondientes a un único ejemplar): una rama mandibular izquierda (5915), una rama mandibular derecha (5916) y un maxilar izquierdo (5917); MACN A 5918-5921 (elementos correspondientes a un único ejemplar): un fragmento de rostro (5918), una rama mandibular derecha y la sínfisis del lado izquierdo (5919); el extremo distal de la ulna (5920) y el extremo distal del húmero (5921) estaban extraviados en las colecciones del MACN al momento de realizar esta revisión. 


\begin{tabular}{|c|c|c|c|c|c|}
\hline MEDIDAS & MLP 11-65 & MLP 11-85 & $\begin{array}{c}\text { MACN } \\
\text { A5915-5017 }\end{array}$ & $\begin{array}{c}\text { MACN } \\
\text { A5918-5921 }\end{array}$ & $\begin{array}{c}\text { CORD-PZ } \\
1210-1 / 5\end{array}$ \\
\hline Longitud anteroposterior del cráneo & 261,2 & - & - & - & 269,2 \\
\hline Longitud del paladar en el plano sagital & $131,2 *$ & - & - & $133 *$ & $135 *$ \\
\hline Ancho máximo del paladar & $124 * *$ & - & - & $134 * *$ & $128,4 * *$ \\
\hline Longitud del canal infraorbitario & - & - & - & 24 & 19 \\
\hline Ancho del hocico a la altura de los caninos & 77 & - & - & $89,3 *$ & $83,4^{* *}$ \\
\hline Ancho del cráneo a la altura del proceso postorbitario & - & - & - & - & 70 \\
\hline Ancho del basicráneo a la altura del meato auditivo externo & 99 & - & - & - & 87,2 \\
\hline Ancho de la cavidad glenoidea & 65,1 & - & - & - & 63,5 \\
\hline Longitud máxima de la cavidad glenoidea & 25,2 & - & - & - & 27,5 \\
\hline Diastema & $10 *$ & - & - & 11 & - \\
\hline Longitud del dentario & 141,5 & - & - & 240 & 210,6 \\
\hline Altura del dentario bajo la raíz posterior del p3 & 46,5 & 53 & 53,5 & 52,7 & 45 \\
\hline Altura del dentario bajo la raíz posterior del $\mathrm{m} 1$ & 47 & 49 & 53 & 51,7 & - \\
\hline Altura del dentario bajo la raíz posterior del $\mathrm{m} 2$ & 47,2 & 47,2 & 52 & 49 & 49,5 \\
\hline Altura del dentario bajo la raíz posterior del $\mathrm{m} 3$ & 50 & 44 & 51 & 49,9 & 51 \\
\hline Altura del dentario bajo la raíz posterior del $\mathrm{m} 4$ & 54,3 & 38,5 & 55 & 51,5 & 56 \\
\hline Longitud de la sínfisis & 69,6 & - & 73,5 & 82,7 & 76 \\
\hline Espacio entre el $\mathrm{m} 4$ y la apófisis coronoides & 15,1 & - & 12 & - & 18,5 \\
\hline Ancho del cóndilo mandibular & - & - & - & + de 52,5 & 58,5 \\
\hline Altura del cóndilo mandibular (labialmente) & 13 & - & - & 14 & 13,5 \\
\hline Altura del cóndilo mandibular (lingualmente) & - & - & - & - & 20 \\
\hline
\end{tabular}

Tabla 2. Medidas craneomandibulares de los ejemplares de Arctodictis munizi considerados en este estudio. Todas las medidas están en milímetros. * indica que la medida es aproximada. ** el valor resulta de multiplicar por dos la dimensión tomada en una de sus mitades.

Measurements of the skull and mandible of the specimens of Arctodictis munizi considered in this study. All the measurements are in millimeters.* shows that is an approached measurement.** the value is the result of multiply by two the dimension taken in one of its parts.

Procedencia geográfica y estratigráfica: Todos los especímenes conocidos de A. munizi proceden de la provincia de Santa Cruz, Argentina, de niveles de la Formación Santa Cruz (Edad Santacrucense, Mioceno Medio). El ejemplar MLP 11-85 proviene de Monte León; los ejemplares MACN A 5915-5917 y 5918-5921 habrían sido exhumados en Corriguen Aike (nivel fosilífero 6) y el ejemplar CORD-PZ 1210-1/5 proviene del Cañadón Las Totoras, Miembro Estancia La Costa, nivel fosilífero 9 (véase más arriba).

\section{Descripción del ejemplar CORD-PZ 1210}

Las estructuras aquí descriptas se ilustran en las figuras 2 a 6; por su parte, las medidas del ejemplar CORD-PZ 1210-1/2 se indican en las tablas 2 a 4. La nomenclatura anatómica fue tomada de Evans y deLahunta (1972) y Muizon (1998). La nomenclatura dentaria sigue a Archer (1976) y Hershkovitz (1982), en este último caso para la homología de los incisivos. El orden adoptado para la descripción es el siguiente: cráneo, dentario, dentición superior, dentición inferior, húmero, tibia y vértebras.

CRÁNEO (Figs. 2a y 3a-c)

El ejemplar CORD-PZ 1210-1 ha conservado casi totalmente la mitad craneana izquierda y buena parte del basicráneo. La región auditiva es fragmentaria, siendo difícil precisar en detalle su estructura.

El premaxilar es angosto, presenta el borde dorsal del proceso facial apenas acuñado entre el extremo anterior del nasal y el anterodorsal del maxilar. La fosa paracanina, definida exclusivamente por el premaxilar, es de desarrollo moderado y carece de una cresta dorsal que la delimite. Sobre el borde anterior de la plataforma narinal se desarrolla una pequeña concavidad; anterior e internamente a ésta, el hueso asciende en forma notoria. $\mathrm{Si}$ bien está roto, dicha morfología sugiere la presencia de pequeños tubérculos óseos cerca del área de contacto entre ambos premaxilares, rasgo presente en otros boriénidos.

La cara lateral del maxilar es más alta que larga, conformando un rostro relativamente corto. Presenta numerosos forámenes nutricios, sobre todo dorsalmente al canino y al foramen infraorbitario. Los yugos alveolares, correspondientes a las raíces de los caninos superiores, están poco marcados. El foramen infraorbitario es grande y abre sobre la raíz anterior del M1. En la región palatina del maxilar abren numerosos forámenes para el pasaje de nervios y vasos al paladar, los cuales son llamados aquí colectivamente forámenes palatinos, y de los cuales el par situado más hacia delante, ubicado a la altura del canino, es el más desarrollado. Lingualmente y en la base de las raíces de los molares superiores, el maxilar presenta numerosos forámenes nutricios de pequeño tamaño. Entre los M3-4, el maxilar desarrolla una profunda fosa para alojar al protocónido del m4, equivalente fosa no existe en los otros espacios intermolares. La plataforma orbitaria está poco extendida y sobre ésta abren algunos forámenes para el pasaje de pequeños nervios y vasos que se dirigen por los conductos alveolares a las raíces de los dientes posteriores (Evans y deLahunta, 1972). El pequeño foramen 


\begin{tabular}{|c|c|c|c|c|}
\hline MEDIDA & MLP 11-65 & $\begin{array}{c}\text { MACN A } \\
5915-5917\end{array}$ & $\begin{array}{c}\text { MACN A } \\
5918-5921\end{array}$ & $\begin{array}{c}\text { CORD-PZ } \\
1210-1 / 5\end{array}$ \\
\hline $\mathrm{I} 1-3$ & - & - & - & - \\
\hline I1-M4 & - & - & - & - \\
\hline C-M4 & - & - & - & 98,5 \\
\hline P1-M4 & - & - & - & 78,3 \\
\hline P1-3 & 30,5 & 25,5 & 32,4 & 29 \\
\hline M1-M4 & - & - & - & 51,7 \\
\hline LI1 & 8,3 & - & - & - \\
\hline AI1 & 3,7 & - & - & - \\
\hline LI2 & 7,1 & - & - & - \\
\hline $\mathrm{AI} 2$ & 3,6 & - & - & - \\
\hline LI3 & - & - & 11,4 & - \\
\hline AI3 & - & - & 6,4 & - \\
\hline $\mathrm{LC}$ & 25,5 & 26,2 & 25,5 & 21 \\
\hline $\mathrm{AC}$ & 19 & 18,4 & 20,5 & 18,5 \\
\hline LP1 & 7,8 & - & 10,3 & 7 \\
\hline AP1 & 4,35 & - & - & 4,5 \\
\hline LP2 & 8,5 & - & 9,6 & 8,5 \\
\hline AP2 & 6,5 & - & 7,3 & 6,5 \\
\hline LP3 & 12 & - & 14,3 & 13 \\
\hline AP3 & 8,5 & - & 10,2 & 9,5 \\
\hline LM1 & - & - & - & - \\
\hline AM1 & - & - & - & - \\
\hline LM2 & - & - & - & 14 \\
\hline AM2 & - & - & - & - \\
\hline LM3 & 17,5 & - & - & 16 \\
\hline AM3 & - & - & - & - \\
\hline LM4 & - & - & - & - \\
\hline AM4 & - & - & - & 12,5 \\
\hline
\end{tabular}

Tabla 3. Medidas de la serie dentaria superior de los ejemplares de Arctodictis munizi considerados en este estudio. L, largo; A, ancho. Todas las medidas están en milímetros.

Measurements of the upper tooth series of the specimens of Arctodictis munizi considered in this study. L, length; $A$, wide. All the measurements are in millimeters.

palatino posterior (posterolateral) está limitado por el maxilar y palatino. En vista ventral, el maxilar se expande lateralmente, formando "mejillas". Dicho carácter constituye una plesiomorfía para los Borhyaenidae (Rougier et al., 1998).

Los nasales son relativamente cortos (casi no sobrepasan el proceso facial del premaxilar) y se ensanchan posteriormente a la altura del contacto con los lacrimales. El borde posterior tiene forma de "W" muy abierta, con la cuña del frontal poco marcada. Existe contacto entre el nasal y el lacrimal, separando por alrededor de $1 \mathrm{~cm}$ el contacto maxilo-frontal. El tubérculo lacrimal está bien desarrollado; el único foramen lacrimal abre internamente en la cavidad orbitaria. Tanto el contacto nasolacrimal como el desarrollo del tubérculo lacrimal, constituyen plesiomorfías para los Borhyaenidae (Rougier et al., 1998).

El borde ventral del yugal posee una zona rugosa, bien delimitada, que constituye el área de origen del músculo masetero profundo (Osgood, 1921). El proceso preglenoideo del yugal está bien desarrollado (en vista lateral su altura es subigual al proceso postglenoideo del escamoso). Las apófisis postorbitarias tanto del yugal como del frontal se encuentran poco marcadas.
Las crestas temporales del frontal están poco definidas, a diferencia de la cresta sagital. En el frontal izquierdo, posteriormente a la apófisis postorbital, abre un pequeño foramen supraorbital. Poterolateralmente este hueso contacta con el escamoso, rasgo presente en otros borieninos (Muizon, 1999).

En la superficie dorsal del cráneo, posteriormente al frontal, se observa un único elemento, de aspecto triangular y poco expandido lateralmente, el cual podría representar los parietales fusionados entre sí, o si osifica, fusionados con el interparietal. Sobre el área más dorsal de la fosa temporal, afectando fundamentalmente el frontal, y los parietal-interparietal se observan numerosas rugosidades que podrían corresponder al área de orígen del músculo temporal (Osgood, 1921).

Los pterigoides no están bien preservados; probablemente estaban poco expuestos en vista lateral (el aliesfenoides y el palatino forman básicamente el área pterigoidea en el sector lateral del cráneo). Ventralmente el pterigoides cubre el aliesfenoides y probablemente la porción distal del presfenoides.

Los límites del orbitoesfenoides no son evidentes. Su límite posterior expuesto estaría delimitado por el borde lateral de la fisura esfenorbital.

El aliesfenoides limita posteriormente a la fisura esfenorbital. Sobre el sector lateral del cráneo, ventral y posteriormente a dicha fisura, abre el foramen rotundo, de considerablemente menor dimensión que la fisura. El foramen oval estaría ubicado ventralmente entre el aliesfenoides y el petroso. No está presente el foramen del canal transverso.

Como ocurre en otros mamíferos, los límites entre el basiesfenoides y el presfenoides no están definidos. Dos robustas crestas se desarrollan como continuación de los bordes laterales engrosados del basioccipital. Lateralmente a las crestas se definen los surcos del tubo de Eustaquio. El foramen carotídeo abre a la altura de la sutura entre el basioccipital y el basiesfenoides; es poco visible en vista ventral ya que está cubierto parcialmente por el basiesfenoides.

El escamoso está bien desarrollado lateral y dorsalmente. La cresta supraglenoidea forma un ángulo casi recto con el arco zigomático. La cavidad glenoidea es de forma suboval, muy ensanchada en su eje latero-medial. El foramen subescamoso abre por encima del meato auditivo externo. El foramen postglenoideo abre sobre el proceso postglenoideo, en una posición medial, de forma tal que no se aprecia en vista posterior al ser cubierto por la superficie occipital.

La región auditiva está mal preservada, por lo que resulta difícil su descripción. El meato auditivo externo está bien circunscrito dorsal, anterior y posteriormente. No hay evidencias de la participación del petroso en el área mastoidea.

El supraoccipital se encuentra fuertemente inclinado hacia atrás, destacándose el gran desarrollo de las crestas lambdoideas, las cuales otorgan un aspecto subcuadrangular a la parte posterior del cráneo. No existe participación del supraoccipital en la delimitación del foramen magno (carácter plesiomórfico para los Borhyaenidae, Rougier et al., 1998).

Los cóndilos occipitales son de morfología oval y salientes posteriormente. Se desarrollan dos forámenes hipoglosos subiguales en tamaño. Los procesos paracondilares están poco desarrollados ventralmente, casi a la misma altura que el escamoso.

Los bordes laterales del basioccipital están bien engrosados y no se aprecia una quilla media. El basioccipital limita posteriormente el foramen del seno petroso inferior. 


\begin{tabular}{|c|c|c|c|c|c|c|c|}
\hline MEDIDAS & MLP 11-65 & MLP 11-81 & MLP 11-85 & MLP 11-89 & $\begin{array}{c}\text { MACN } \\
\text { A5915-17 }\end{array}$ & $\begin{array}{c}\text { MACN } \\
\text { A5918-21 }\end{array}$ & $\begin{array}{c}\text { CORD-PZ } \\
1210-1 / 5\end{array}$ \\
\hline i1-3 & - & - & - & - & $16^{*}$ & $12,5^{*}$ & 16 \\
\hline i1-m4 & - & - & - & - & 126 & - & 111,3 \\
\hline $\mathrm{c}-\mathrm{m} 4$ & 119 & - & 116,5 & - & 126 & - & 111,3 \\
\hline p1-m4 & - & - & 100 & - & 92,3 & - & 85 \\
\hline p1-3 & - & - & 33,3 & - & 29 & 34,4 & $28 *$ \\
\hline m1-m4 & 59 & - & 61,5 & - & 62,7 & 64,5 & - \\
\hline Li1 & 6 & - & - & - & - & - & 7,2 \\
\hline Ai1 & 3,1 & - & - & - & - & - & 3 \\
\hline $\mathrm{Li} 2$ & 5,5 & - & - & - & - & - & 6,5 \\
\hline Ai2 & 3,9 & - & - & - & - & - & 2,7 \\
\hline Li3 & - & - & - & - & - & - & 6,2 \\
\hline Ai3 & - & - & - & - & - & - & 5 \\
\hline $\mathrm{Lc}$ & 23,8 & - & - & - & 26 & 22,8 & 24 \\
\hline Ac & 16,5 & - & - & - & 17 & 17,5 & 13 \\
\hline Lp1 & - & - & 13 & - & - & - & 12,5 \\
\hline Ap1 & - & - & 5 & - & - & - & 5 \\
\hline Lp2 & 12 & - & 12,3 & - & 11,8 & - & 10 \\
\hline Ap2 & 7,2 & - & 8 & - & 8,4 & 9 & 6,5 \\
\hline Lp3 & - & - & 12,2 & - & 13,4 & 14,4 & - \\
\hline Ap3 & - & - & 8,2 & - & 8,4 & 9,5 & - \\
\hline Lm1 & 13,2 & - & 12 & - & 12,8 & - & - \\
\hline Am1 & 7,5 & - & 7 & - & 8 & 8,9 & - \\
\hline $\mathrm{Lm} 2$ & 13,2 & - & 13,8 & 14,1 & 16,3 & 14,5 & 13 \\
\hline $\mathrm{Am} 2$ & 8 & - & 8,5 & 8,5 & 11 & 11 & 8 \\
\hline Lm3 & 15 & - & 17,5 & - & - & 17,3 & - \\
\hline Am3 & 9,5 & - & 10 & - & - & 11,7 & - \\
\hline Lm4 & 19,2 & 18,5 & 19 & - & - & 21,6 & - \\
\hline Am4 & 10,5 & 9,6 & - & - & - & 13,5 & 11,5 \\
\hline
\end{tabular}

Tabla 4. Medidas de la serie dentaria inferior de los ejemplares de Arctodictis munizi considerados en este estudio. L, largo; A, ancho. Todas las medidas están en milímetros. * indica que la medida es aproximada.

Measurements of the lower tooth series of the specimens of Arctodictis munizi considered in this study. L, length; A, wide. All the measurements are in millimeters.* shows that is an approached measurement.

\section{Dentario (Figs. 2a y 3d)}

Se ha conservado la hemimandíbula izquierda y la región sinfisaria de la hemimandíbula derecha fusionada. El dentario es un hueso robusto, con el borde ventral recto hasta aproximadamente el límite de los p2-p3. El extremo anterior del cuerpo mandibular es casi vertical, implantándose los incisivos por detrás de la cara anterior de ambos caninos (excepto los i4, que se implantan algo más anteriormente).

La sínfisis mandibular se extiende posteriormente hasta la altura del p3-m1. Sobre la cara anterior del cuerpo mandibular, por debajo de los incisivos y a ambos lados de la sínfisis, se aprecian varios forámenes nutricios, algunos de gran tamaño (hasta $3 \mathrm{~mm}$ de diámetro). Sobre el borde ventral de cada hemimandíbula, cerca de la región sinfisaria, se desarrollan depresiones alargadas, casi paralelas al eje mandibular, que posiblemente constituyen las áreas de origen del músculo digástrico.

Sobre la superficie labial de la rama horizontal del dentario abren cuatro forámenes mentonianos. El primero de ellos es el de mayor tamaño y abre bajo la raíz anterior del p2. Los restantes son subiguales entre sí y de tamaño considerablemente menor. De adelante hacia atrás, los forámenes se ubican bajo la raíz posterior del p3, bajo el m1 y bajo la raíz anterior del m2.
Los forámenes segundo y tercero abren a la altura del borde superior del primero, mientras que el cuarto lo hace algo más dorsalmente aún.

Sobre la superficie lingual de la rama horizontal del dentario, el surco mandibular está muy poco marcado y es relativamente corto. El foramen dentario abre por debajo y posteriormente a la base de la cresta coronoidea anterior.

El proceso coronoides está bien desarrollado, con sus bordes anterior y posterior suavemente curvados posteriormente. La fosa masetérica, sobre la cara labial, es amplia y llega casi hasta la altura del borde posterior del $\mathrm{m} 4$. La cresta coronoidea anterior delimita externamente un surco profundo que corre por debajo y detrás del $\mathrm{m} 4$. En vista medial, en la base del proceso coronoides, por debajo del $\mathrm{m} 4$ se desarrolla una amplia superficie oval de aspecto rugoso, que podría corresponder con la inserción distal de algún tendón del músculo temporal. El proceso angular está bien curvado medialmente y define una amplia superficie para la inserción de la musculatura pterigoidea (Hiiemae y Jenkins, 1969). El cóndilo mandibular está bien desarrollado transversalmente, estando a su vez más engrosado lingualmente que labialmente. La unión de la cresta infracondilar con la rama horizontal describe por su parte una suave curva. 

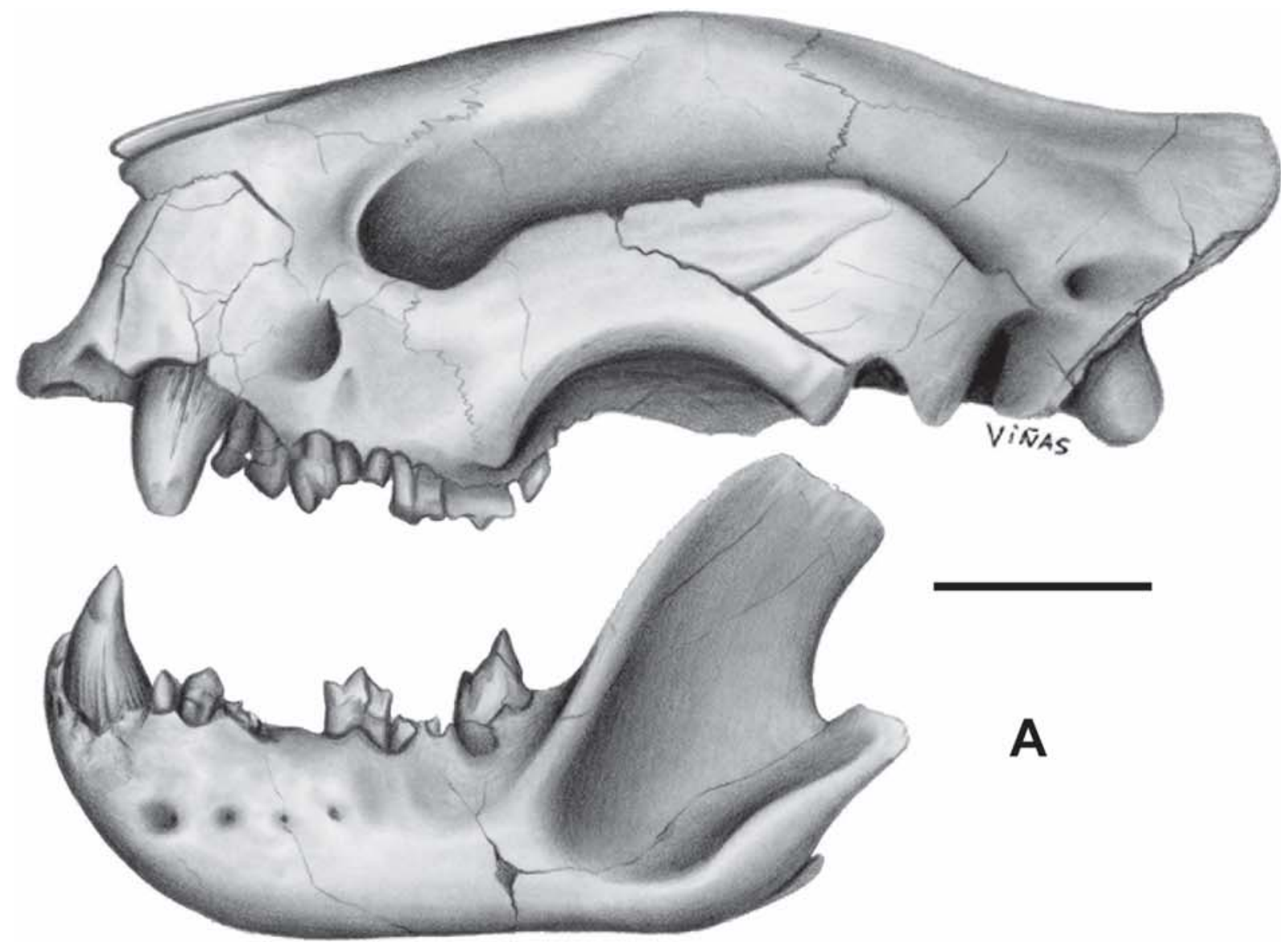

A
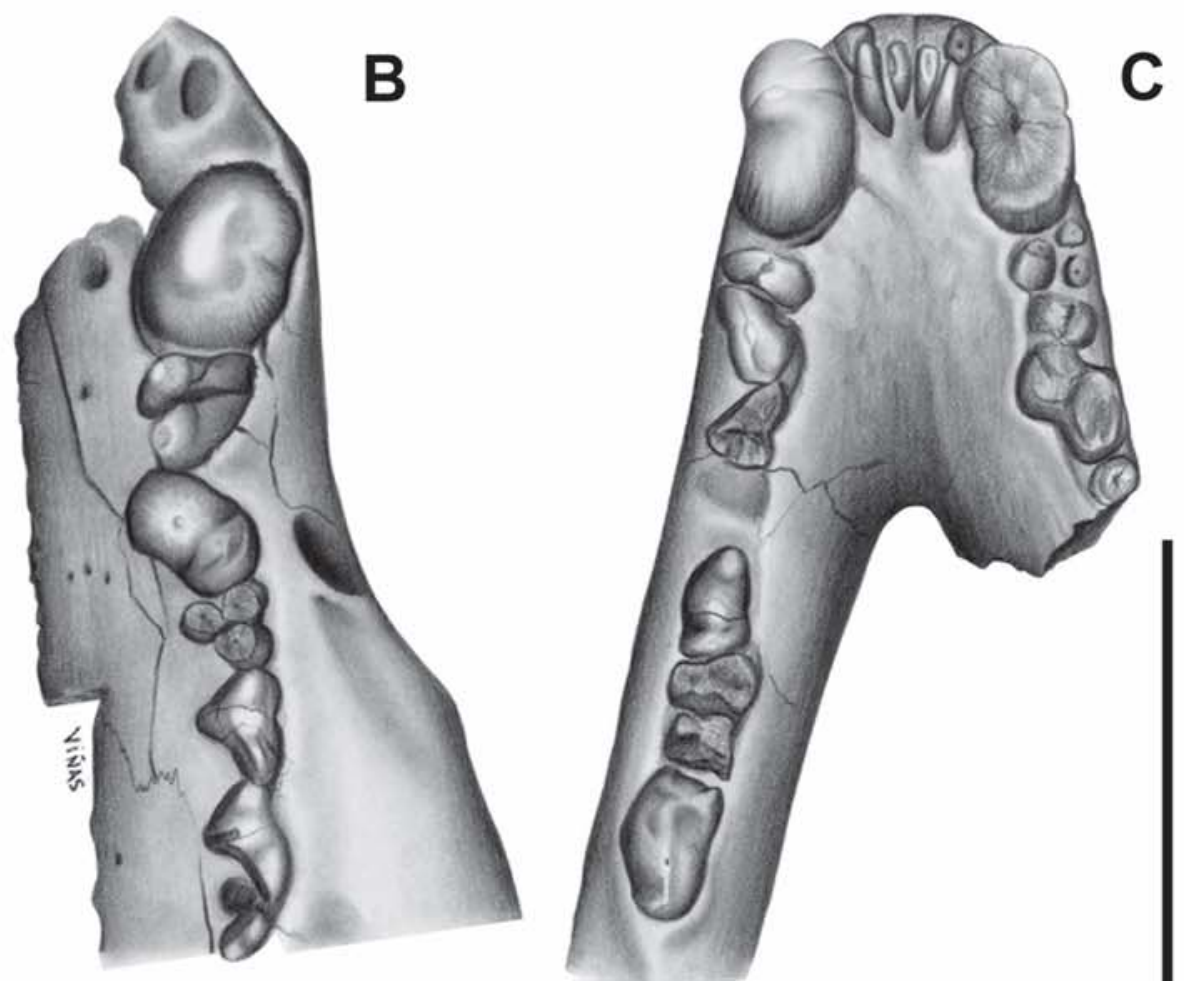

Figura 2. Arctodictis munizi Mercerat, 1891. Cráneo y mandíbula del ejemplar CORD-PZ 1210-1/2 en vista lateral (A), y detalle de la dentición superior (B) e inferior (C) en vista oclusal. Escala: $5 \mathrm{~cm}$.

Arctodictis munizi Mercerat, 1891. Skull and mandible of the specimen CORD-PZ 1210-1/2 in lateral view (A), and detail of the upper teeth $(B)$ and lower teeth $(C)$ in occlusal view. Scale: $5 \mathrm{~cm}$. 

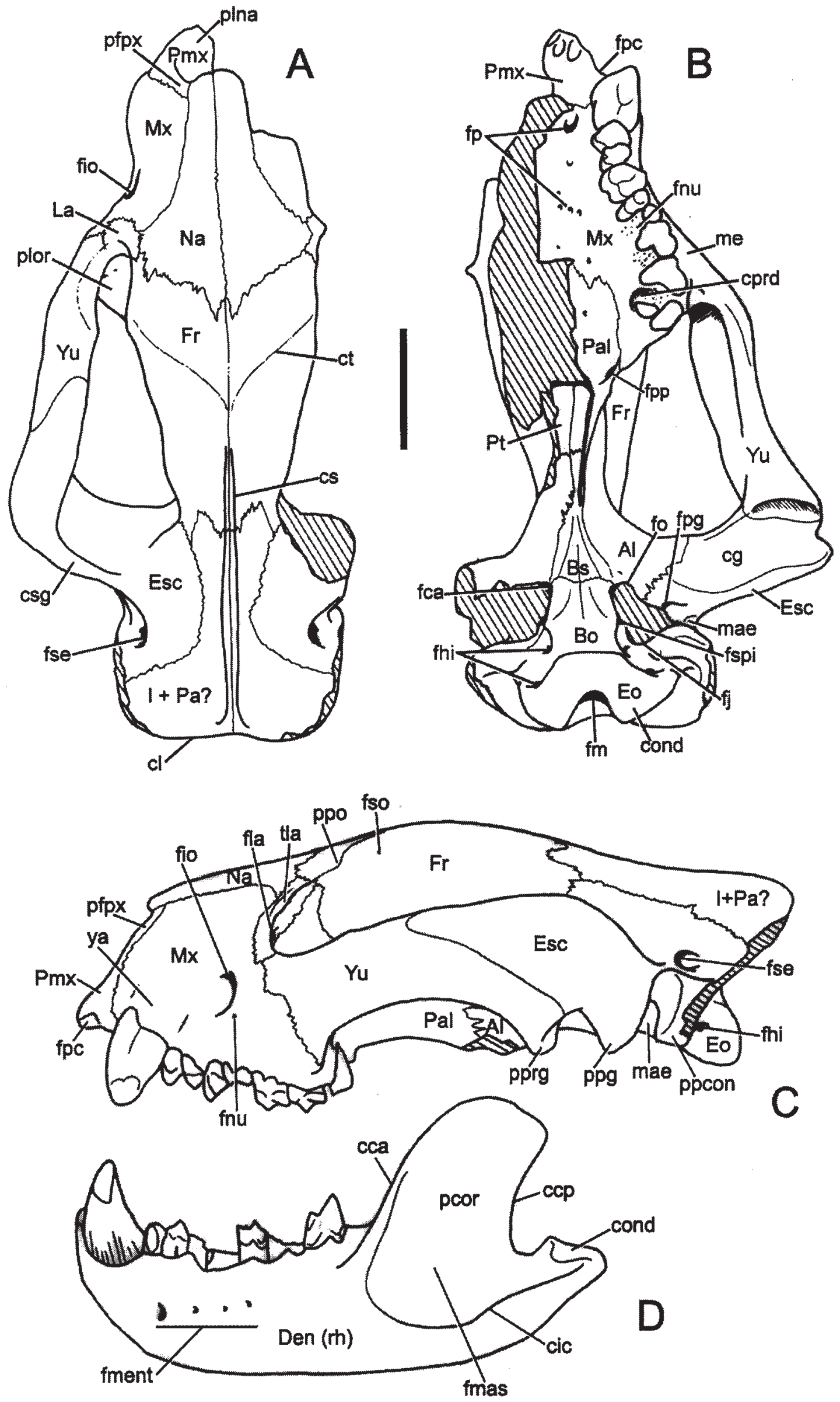


\section{Dentición superior (Fig. 2b)}

El ejemplar presenta tres incisivos de los que sólo se han conservado las raíces de los I2-3 y el alvéolo en el I4. La dimensión del alvéolo del I4 permite inferir un mayor tamaño. El I2 estaba algo más adelantado que los I3-4, a juzgar por la raíz y el alvéolo preservados.

Los caninos son muy robustos, marcados dorsoventralmente por dos surcos principales, uno lateral y otro medial. Sobre la base de cada canino se aprecian numerosos surcos de escasa profundidad, paralelos entre sí y a los primeros. La cubierta de esmalte está restringida a la región apical del diente.

Los tres premolares son birradiculados y se imbrican entre sí. No hay diastema entre el canino y P1 ni entre los premolares. El P1 se implanta transversal sobre el eje dentario; el P2 lo hace casi transversalmente, mientras que el P3 se dispone en forma oblicua aunque inversamente a los anteriores, ocupando la raíz anterior una posición más lingual y la posterior más labial. La corona del P2 es algo mayor que la del P1; ambas muestran una cúspide principal central y crestas en las laderas anterolabiales y posterolinguales. En ambos premolares, las coronas no se orientan según el eje delimitado por ambas raíces sino que están levemente torsionadas. El P3 es mucho más grande que los premolares anteriores; sobre el cíngulo posterior, más extendido sobre la cara lingual que sobre la labial, se desarrolla una cúspide globosa de posición postero-labial. Por detrás y labialmente a la cúspide principal, se desarrolla una corta cresta, no así sobre la cara anterior, presente en los otros premolares.

De los cuatro molares presentes originalmente, sólo se han preservado los M2-4 completos aunque muy desgastados y las tres raíces del M1. El M2 es claramente menor que el M3. En los M2-3 la raíz posterolateral aumenta progresivamente de tamaño hacia atrás; el ectoflexo está muy poco definido, el paracono es apenas distinguible y coalescente con la ladera anterior del metacono; el protocono es vestigial o ausente. El parastilo es pequeño pero distinguible en ambos dientes. El metacono ocupa una posición central en cada diente y la postmetacrista está notablemente desarrollada. El M4 es pequeño aunque no vestigial; presenta dos raíces casi coalescentes y está fuertemente desgastado.

\section{Dentición inferior (Fig. 2c)}

Presenta tres incisivos, subigales entre sí y fuertemente comprimidos lateralmente. Con la excepción del i4, se encuentran implantados a la altura del borde anterior del canino. El i3 está fuertemente retrasado, siendo el carácter "sobremontado" (staggered; véase Hershkovitz, 1982) claramente distinguible. Por su parte, e inusualmente, el i4 se implanta muy por delante del i2, ubicándose el borde posterior al mismo nivel que el borde anterior del i2.

Sólo se ha preservado el canino izquierdo; es robusto y de sección oval. Su base muestra numerosos surcos paralelos al eje principal del diente, destacándose uno sobre la cara lateral y dos sobre la cara medial. El esmalte está restringido al tercio apical del diente. Sobre su cara posterolateral, una fuerte faceta de desgaste ha dejado expuesta la dentina.

De los tres premolares, sólo se han preservado los p1-2 izquierdos, del p3 sólo se conservan las raíces. Toda la serie premolar está fuertemente imbricada, con sus dos raíces globosas y expuestas. En el p1 las raíces son subiguales entre sí y se ubican transversalmente al eje dentario. En el p2 las raíces, también subiguales, se ubican en sentido anterolingual-posterolateral. La corona posee una cúspide principal asimétrica, algo desplazada hacia el extremo anterior del diente. Tanto por delante como por detrás de la cúspide principal se desarrollan

Figura 3. Arctodictis munizi Mercerat, 1891. Cráneo del ejemplar CORD-PZ 1210-1/2 en vista dorsal (A), ventral (B) y lateral (C) y mandíbula en vista lateral (D). Abreviaturas. Al: Aliesfenoides; Bo: Basioccipital; Bs: Basiesfenoides; cca: cresta coronoidea anterior; ccp: cresta coronoidea posterior; cg: cavidad glenoidea; cic: cresta infracondilar; cl: cresta lambdoidea; cond: cóndilo; cprd: concavidad del protocónido sobre el maxilar; cs: cresta sagital; csg: cresta supraglenoidea; ct: cresta temporal; Den (rh): rama horizontal del dentario; Eo: Exoccipital; Esc: Escamoso; fca: foramen carotídeo; fhi: foramen hipogloso; fio: foramen infraorbitario; fj: foramen yugular; fla: foramen lacrimal; fm: foramen magno; fmas: fosa masetérica; fment: foramen mentoniano; fnu: foramen nutricio; fo: foramen oval; fp: foramenes palatinos; fpc: fosa paracanina; fpg: foramen postglenoideo; fpp: foramen palatino posterior; Fr: Frontal; fse: foramen subescamoso; fso: foramen supraorbital; fspi: foramen del seno petrosal inferior; I+Pa?: ¿interparietal y parietal fusionados?; La: Lacrimal; mae: meato auditivo externo; me: "mejillas" del maxilar; Mx: Maxilar; Na: Nasal; Pal: Palatino; pcor: proceso coronoides; pfpx: proceso facial del premaxilar; plna: plataforma narial; plor: plataforma orbitaria; Pmx: Premaxilar; ppcon: proceso paracondilar; ppg: proceso postglenoideo; ppo: proceso postorbital; pprg: proceso preglenoideo; Pt: Pterigoides; tla: tubérculo lacrimal; ya: yugos alveolares; Yu: Yugal. Escala: $5 \mathrm{~cm}$.

Arctodictis munizi Mercerat, 1891. Skull of the specimen CORD-PZ 1210-1/2 in dorsal (A), ventral (B) and lateral (C) views and mandible in lateral view (D). Abbreviations. Al: Alisphenoid; Bo: Basioccipital; Bs: Basisphenoid; cca: anterior coronoid crest; ccp: posterior coronoid crest; cg: glenoid cavity; cic: infracondylar crest; cl: lambdoid crest; cond: condyle; cprd: protoconid pit in the maxilla; cs: sagittal crest; csg: supraglenoid crest; ct: temporal crest; Den (rh): horizontal ramus of the dentary; Eo: Exoccipital; Esc: Squamosal; fca: carotid foramen; fhi: hypoglosal foramen; fio: infraorbital foramen; fj: jugular foramen; fla: lacrimal foramen; fm: foramen magnum; fmas: masseteric fossa; fment: mental foramina; fnu: nutrient foramen; fo: foramen oval; fp: palatine foramina; fpc: paracanine fossa; fpg: postglenoid foramen; fpp: posterior palatine foramen; Fr: Frontal; fse: subsquamosal foramen; fso: supraorbital foramen; fspi: foramen for the inferior petrosal sinus; I+Pa?: interparietal and parietal fused?; La: Lacrimal; mae: external acoustic meatus; me: "cheeks" of the maxilla; Mx: Maxilla; Na: Nasal; Pal: Palatine; pcor: coronoid process; pfpx: facial process of the premaxilla; plna: narial platform; plor: orbital platform; Pmx: Premaxilla; ppcon: paracondylar process; ppg: postglenoid process; ppo: postorbital process; pprg: preglenoid process; Pt: Pterigoid; tla: lacrimal tubercle; ya: alveolar bulk; Yu: jugal. Scale: $5 \mathrm{~cm}$. 
crestas, estando la posterior algo más marcada que la anterior. En el p3 la raíz posterior está mucho más desarrollada que la anterior; el sentido de implantación de las mismas es anterolingual-posterolabial, esto es, en forma inversa a la implantación de los p1-2.

De los cuatro molares originalmente presentes sólo se han conservado los $\mathrm{m} 2$ y m4 izquierdo (del $\mathrm{m} 3$ sólo se conservan las raíces). Todos los molares tienen sus dos raíces globosas y expuestas. La raíz anterior aumenta proporcionalmente de tamaño hacia el m4. En el $\mathrm{m} 2$ las cúspides del trigónido están muy desgastadas; sobre la ladera posterior del protocónido se aprecia una pequeña cresta. El talónido es mucho más ancho que largo e incluye una pequeña cúspide de incierta homología (véase más abajo). La cara labial del talónido es mucho más vertical que la lingual. Sobre la cara labial se aprecia que un lóbulo de la corona se extiende sobre la raíz posterior claramente más ventralmente que el lóbulo anterior. El m4 es mucho más grande que el $\mathrm{m} 2$ y prácticamente carece de talónido. El protocónido desarrolla, además de la preprotocrístida, una muy marcada postprotocrístida.

\section{Homología de la cúspide posterobasal de los molares inferiores}

La homología de la cúspula posterobasal de los molares inferiores, tanto de Arctodictis como de los Borhyaeninae en general, es problemática. En el ejemplar FMNH P13526 de Arctodictis sinclairi, se aprecia con claridad la base de dicha cúspula por detrás y algo lingualmente al protocónido del $\mathrm{m} 2$. Inmediatamente por detrás de esta cúspula, se observa un cíngulo muy comprimido anteroposteriormente, que podría corresponder al talónido vestigial. La cuestión a resolver es si la cúspula se implanta sobre este talónido vestigial o si, por el contrario, lo hace sobre la ladera posterior del protocónido. En el primer caso, sería discutible su homología con el entocónido o, más probablemente, el hipocónido. En el segundo caso, la cúspula debería homologarse con un metacónido de carácter vestigial.

Marshall (1978) consideró que los boriénidos postdeseadenses pierden el metacónido, carácter presente en los boriénidos más antiguos; no obstante, la presencia de la cúspide mencionada en el párrafo precedente y su dificultad para establecer homologías sugieren la realización de un análisis más detallado del carácter en un contexto filogenético.

\section{POSTCRÁNEO}

Húmero (Fig. 4a). Se ha conservado parcialmente el extremo proximal del húmero derecho (CORD-PZ 1210-3), aunque bastante comprimido antero-posteriormente, posiblemente debido a procesos tafonómicos (véase más arriba). El sector preservado de la diáfisis es recto; el extremo más distal preservado es relativamente angosto. La cabeza es esferoidal; debajo y sobre el margen posteromedial, se desarrolla una concavidad que constituye el área de origen del músculo triceps brachial caput mediale (Argot, 2001); ésta se encuentra comparativamente menos desarrollada que en otros borienidos (Fig. 5a). El tubérculo menor está muy poco desarrollado; no obstante, el área de

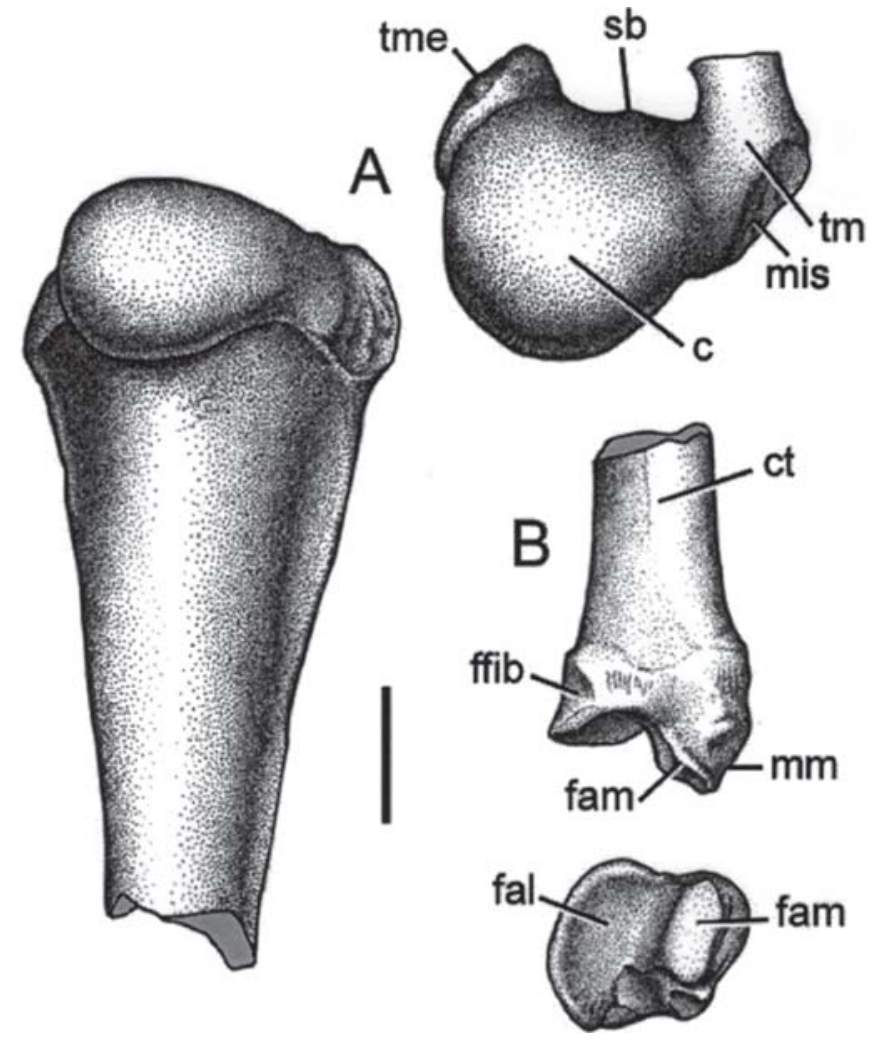

Figura 4. Arctodictis munizi Mercerat, 1891. Fragmento proximal del húmero derecho del ejemplar CORDPZ 1210-3 en vista proximal y posterior (A) y fragmento distal de la tibia derecha del mismo ejemplar en vista anterior y distal (B). Abreviaturas: c: cabeza; ct: cresta tibial; fal: faceta astragaliana lateral; fam: faceta astragaliana medial; ffib: faceta fibular; mis: faceta de inserción del músculo infraespinoso; mm: maléolo medial; sb: surco bicipital; tm: tubérculo mayor; tme: tubérculo menor. Escala: $2 \mathrm{~cm}$. Arctodictis munizi Mercerat, 1891. Proximal portion of the right humerus of the specimen CORD-PZ 1210-3 in proximal and posterior views (A) and distal portion of the right tibia of the same specimen in anterior and distal views (B). Abbreviations: $c$ : head; ct: tibial crest; fal: lateral astragalar facet; fam: medial astragalar facet; ffib: fibular facet; mis: infraspinatus facet; $\mathrm{mm}$ : medial malleolus; $s b$ : bicipital groove; tm: mayor tubercle; tme: lesser tubercle. Scale: $2 \mathrm{~cm}$.

inserción del músculo subescapular es amplia y de forma oval, con el eje mayor orientado en sentido anterodorsal-posteroventral. El surco bicipital es amplio, aunque aparentemente se encuentra afectado por la deformación. La cresta tricipital parece haber estado bien marcada (aunque por efecto de la compresión del material, dicho carácter está algo exagerado). Tanto el tubérculo mayor como la cresta deltoidea están parcialmente preservados. Aparentemente, y al igual que en otros borieninos, el tubérculo mayor se extiende hasta casi la misma altura que la cabeza (en Prothylacynus, al contrario, apenas la sobrepasa; Fig. 5a). Sobre la cara posterolateral del tubérculo mayor existe una 
superficie oval bien delimitada, presente también en otros boriénidos, donde se insertaría el músculo infraespinoso (Argot, 2001).

Tibia (Fig. 4b). Se ha conservado el fragmento distal de la tibia derecha (CORD-PZ 1210-4). En este sector, la diáfisis está algo comprimida lateralmente; la cresta tibial está poco marcada. La faceta articular astragaliana se divide en dos sectores: lateral y medial, con una ángulo de casi $90^{\circ}$ entre ambos. La faceta lateral es plana, reniforme, inclinada lateralmente y algo más angosta que en otros borienidos (Fig. 5b). La faceta medial se extiende sobre el maléolo y se distinguen dos superficies articulares: una netamente medial y otra ventromedial, con un ángulo entre sí de aproximadamente $135^{\circ}$. El maléolo está bien desarrollado. Sobre el sector lateral y distal de la epífisis, se desarrolla una clara faceta para la articulación de la fíbula.

Vértebras (Fig. 6). Se han preservado dos vértebras (CORDPZ 1210-5), una torácica proximal (Fig. 6a) y otra caudal proximal (Fig. 6b). El cuerpo vertebral de la primera de ellas es robusto, con una marcada cresta ventral (hipapófisis); a ambos lados de dicha cresta abren dos forámenes nutricios. Las caras articulares son de forma triangular, dado el desarrollo de la faceta de articulación para el capitulum costal. El canal neural es amplio y de forma oval. Las superficies articulares de las
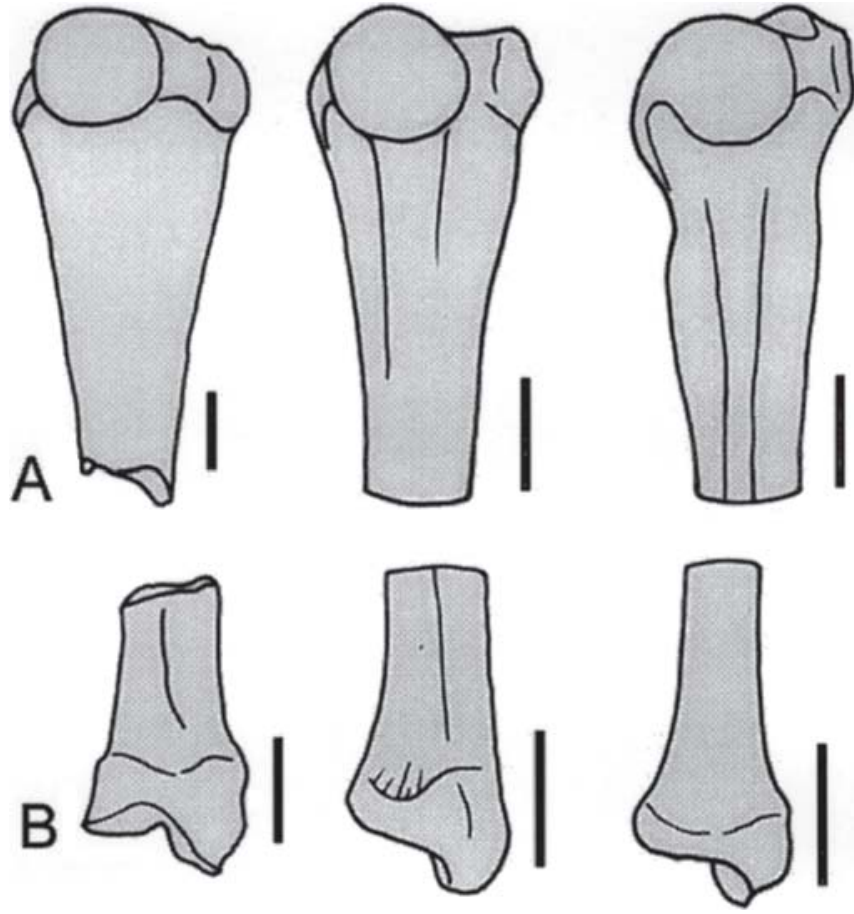

Figura 5. Dibujo lineal comparativo de Arctodictis munizi, A. sinclairi y Prothylacynus patagonicus (de izquierda a derecha). Porción proximal del húmero en vista posterior (A) y porción distal de la tibia en vista anterior (B). Escala: $2 \mathrm{~cm}$.

Technical drawing comparing Arctodictis munizi, A. sinclairi and Prothylacynus patagonicus (from left to right). Proximal portion of the humerus in posterior view $(A)$ and distal portion of the tibia in anterior view (B). Scale: $2 \mathrm{~cm}$. prezigapófisis se inclinan ventromedialmente, condición presente en la segunda vértebra torácica de otros boriénidos. Los pedicelos son cortos y tanto la escotadura vertebral anterior como la posterior están marcadas, si bien la segunda es de considerablamente mayor desarrollo. Los procesos transversos son cortos, robustos y están dirigidos horizontalmente; sobre ellos se desarrolla una cresta. La faceta para la articulación del tuberculum costal es amplia, de forma oval y ubicada casi transversal al eje del proceso transverso. La espina neural está inclinada suavemente hacia atrás y, al igual que en otros boriénidos, presenta posteriormente una cresta.

A juzgar por la morfología del arco neural y la posición del proceso transverso, la vértebra caudal preservada corresponde a una proximal (segunda, tercera o cuarta). El cuerpo vertebral presenta ventralmente dos pares de crestas, paralelas entre sí, sobre el margen anterior y posterior; el par posterior está algo más desarrollado que el anterior. Los procesos transversos se originan a la altura del borde dorsal del cuerpo vertebral. El arco neural está inclinado posteriormente y sostiene una espina neural muy poco desarrollada. Las prezigapófisis se orientan dorsomedialmente y sobre ellas se desarrollan las metapófisis. Las postzigapófisis están separadas entre sí y su faceta se orienta ventrolateralmente.

\section{COMPARACIONES CON EL HOLOTIPO Y EL MATERIAL REFERIDO}

MLP 11-85 (tipo de Arctodictis munizi Mercerat, 1891; Fig. 7), un fragmento mandibular izquierdo con la raíz del canino y los p1-m4 (este último roto en su mitad posterior) y el sector sinfisario de la rama mandibular derecha, con las raíces del canino y los p1-2 y el p3 casi completo.

La mandíbula del ejemplar MLP 11-85 es más robusta que aquella del CORD-PZ 1210-2. A diferencia de este último, en el MLP 11-85 el segundo foramen mentoniano en la hemimandíbula izquierda es el de mayor tamaño, mientras que en la derecha está dividido dos. Las raíces de los dientes postcaninos son más globosas que en el CORD-PZ 1210-2. El p2 presenta el cíngulo posterolingual algo más desarrollado. Al menos en los m1-2, sobre el cíngulo labial se desarrollan pequeñas cúspulas poco notorias; este carácter no es observable en el ejemplar CORD-PZ 1210-2, si bien sólo es confrontable en el m2.

MLP 11-65, un cráneo incompleto y reconstruido con la mandíbula asociada.

La mandíbula de este ejemplar es algo más larga y alta, con el espacio entre el $\mathrm{m} 4$ y el proceso coronoides más amplio que el ejemplar CORD-PZ 1210-2. Este carácter podría relacionarse con la edad; los individuos adultos tienden a incrementar la distancia entre el $\mathrm{m} 4$ y el proceso coronoides. El proceso angular es algo más robusto. El cráneo del ejemplar MLP 11-65 se encuentra en gran parte reconstruido, por lo que resulta difícil su interpretación anatómica. Es algo más robusto que el CORD-PZ 1210- 

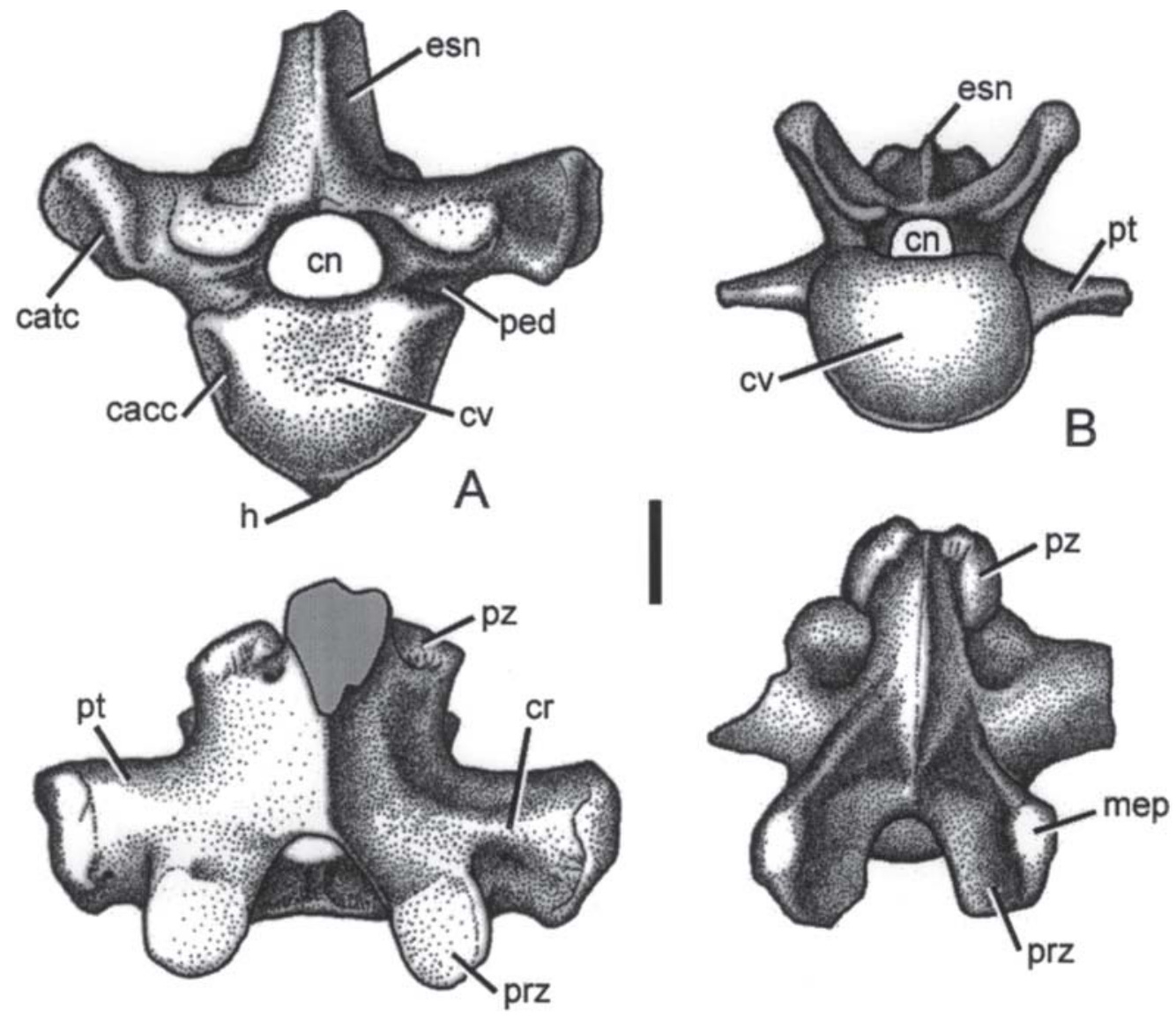

Figura 6. Arctodictis munizi Mercerat, 1891. Vértebra torácica proximal del ejemplar CORD-PZ 1210-5 en vista anterior y dorsal (A) y vértebra caudal proximal del ejemplar CORD-PZ 1210-6 en vista anterior y dorsal (B). Abreviaturas: cacc: carilla articular para el capitulum costal; catc: carilla articular para el tuberculum costal; cn: canal neural; cr: cresta; cv: cuerpo vertebral; esn: espina neural; h: hipapófisis; mep: metapófisis; ped: pedicelo; prz: prezigapófisis; pt: proceso transverso; pz: postzigapófisis. Escala: $1 \mathrm{~cm}$.

Arctodictis munizi Mercerat, 1891. Proximal thoracic vertebra of the specimen CORD-PZ 1210-5 in anterior and dorsal views (A) and proximal caudal vertebra of the specimen CORD-PZ 1210-6 in anterior and dorsal views (B). Abbreviations: cacc: articular surface for the head of the rib; catc: articular surface for the tubercle of the rib; cn: neural canal; cr: crest; cv: vertebral body; esn: neural spine; $h$ : hipapophysis; mep: metapophysis; ped: pedicle; prz: prezygapophysis; pt transverse process; pz: postzygapohysis. Scale: $1 \mathrm{~cm}$.

1, con las crestas lambdoideas algo más desarrolladas. En vista ventral, se aprecia que las "mejillas" resultan más notorias que en el CORD-PZ 1210-1 y que, a la altura del plano sagital, el paladar secundario se extiende claramente por detrás del M4 (este carácter también estaría presente en CORD-PZ 1210-1 aunque resulta más claro en el MLP 11-65 por el estado de preservación del material en este sector). Los caninos presentan pequeños surcos en su base, aunque no tan numerosos y notorios como en el CORDPZ 1210-1/2. En los molares inferiores no se desarrollan cúspulas sobre el cíngulo labial como en el MLP 11-85. Como fue señalado por Cabrera (1927: 809) este ejemplar presenta "...la anomalía de carecer de M4 derecho, particularidad que no parece ser muy rara entre los boriénidos". Sinclair (1906: 392) menciona un caso análogo en el tipo de Cladosictis pattersoni.
MACN A 5915-5917, una rama mandibular izquierda (5915), una derecha (5916) y un maxilar izquierdo (5917).

Se trata de un ejemplar algo más robusto que el CORD-PZ 1210-1/5. La rama mandibular está aún más engrosada por debajo del $\mathrm{m} 4$; la fosa masetérica es algo más profunda que aquella del CORD-PZ 1210-2. Si bien presenta cuatro forámenes mentonianos, con el anterior más desarrollado, la ubicación de los mismos difiere en ambas hemimandíbulas y con aquella del CORD-PZ 12102. En la hemimandíbula izquierda, el primer foramen abre por debajo del p1; el segundo, entre el p3-m1; el tercero, bajo la raíz posterior del $\mathrm{m} 1$ y el cuarto, bajo la raíz posterior del m2. En la hemimandíbula derecha, los forámenes son subiguales excepto el segundo que es algo menor. El primer foramen (del que sólo se ha preservado el sector posterior) abre a la altura del $\mathrm{p} 1$; el segundo lo hace a la 

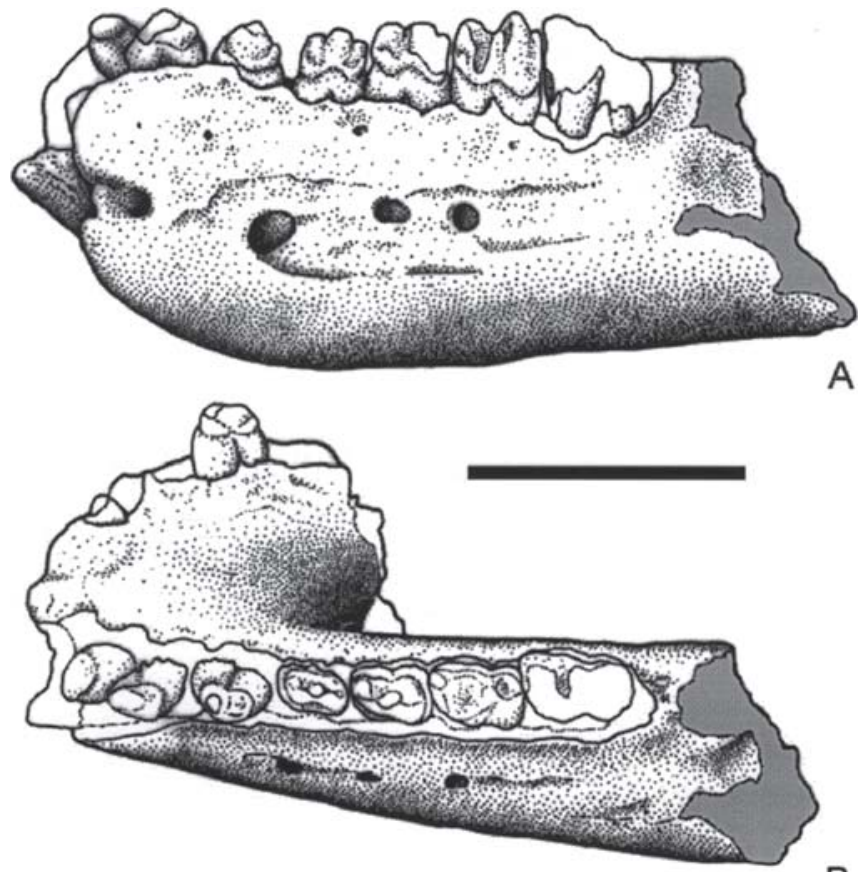

B

Figura 7. Arctodictis munizi Mercerat, 1891. Mandíbula del ejemplar MLP 11-85 (tipo) en vistas labial (A) y oclusal (B). Escala $5 \mathrm{~cm}$.

Arctodictis munizi Mercerat, 1891. Mandible of the specimen MLP 11-85 (type) in labial (A) and occlusal (B) views. Scale: $5 \mathrm{~cm}$.

altura de la raíz posterior del p2; el tercero, bajo la raíz posterior del $\mathrm{m} 1$ y el cuarto, entre el $\mathrm{m} 2$ y $\mathrm{m} 3$. El surco mandibular y las depresiones para el músculo digástrico están algo más marcados que en el CORD-PZ 1210-2, no así la región oval por delante del foramen dentario, para la posible inserción del músculo temporal.

A diferencia del CORD-PZ 1210-2, el p2 presenta una pequeña cúspula anterior a la cúspide principal. Sobre el talónido del m1-2, medialmente a su cúspide principal, se desarrolla una pequeña cúspula.

A diferencia del ejemplar CORD-PZ 1210-1, el foramen infraorbitario es de mayor tamaño, los yugos alveolares correspondientes a los caninos superiores están más marcados y las raíces del P3 están implantadas transversalmente al eje de la hilera dentaria.

MACN A 5918-5921, un fragmento de rostro (5918), una rama mandibular derecha y la sínfisis del lado izquierdo (5919). Los materiales 5920 y 5921 (extremo distal de la ulna y extremo distal de húmero, respectivamente) no se hallaban en las colecciones del MACN al momento de realizar esta revisión.

Después del MLP 11-85, este ejemplar es uno de los más robustos conocidos para esta especie. A diferencia del resto, muestra un marcado incremento en la altura de la mandíbula hacia el extremo anterior. La disposición, nú- mero y tamaño de los forámenes mentonianos es diferente a la del resto de los ejemplares preservados. Hay cinco forámenes mentonianos, de los cuales el cuarto es el mayor, luego el primero y por último el resto, siendo éstos subiguales entre sí. El primer foramen abre por debajo de la raíz anterior del p2; el segundo, bajo la raíz posterior del p2; el tercero, bajo la raíz anterior del p3; el cuarto, bajo el $\mathrm{m} 1$ y el quinto, bajo la raíz posterior del $\mathrm{m} 2$. Sobre el paladar se aprecian numerosas rugosidades y estriaciones óseas de forma irregular.

Son varias las diferencias entre este ejemplar y el CORD-PZ 1210-2: en el dentario el surco mandibular está bien marcado, existe un engrosamiento en la mandíbula debajo del $\mathrm{m} 4$, y la fosa masetérica es muy profunda. En los dientes inferiores, las cúspulas basales de la corona de los premolares y molares no son evidentes y el m4 presenta un pequeño talónido posterior dividido por la postprotocrístida. Por último, en el cráneo, el foramen palatino posterior está bien desarrollado y los yugos alveolares correspondientes a los caninos superiores están bien marcados, desarrollándose sobre ellos numerosos forámenes nutricios.

MLP 11-89, un $m 2$ derecho.

A diferencia del ejemplar CORD-PZ 1210-2, la raíz anterior es de sección circular y más reducida que la posterior, de sección oval y comprimida anteroposteriormente; sobre el cíngulo labial, en la base de la corona, y sobre el cíngulo posterior que rodea al talónido se desarrollan pequeñas y numerosas cúspulas; el cíngulo anterobasal está poco desarrollado.

MLP 11-81, un $\mathrm{m} 4$ izquierdo.

Al igual que en el ejemplar CORD-PZ 1210-2, la raíz anterior del m4 está más desarrollada que la posterior, llegando hasta la mitad del protocónido.

\section{SÍNTESIS}

La mayor parte de las diferencias observadas entre los ejemplares comparados se relacionan con las diferencias en el tamaño y robustez de los mismos: engrosamiento del cuerpo mandibular por debajo del $\mathrm{m} 4$, profundidad en la fosa masetérica, altura del cuerpo mandibular, globosidad de las raíces de los dientes postcaninos, grado de imbricación de las raíces de los postcaninos, desarrollo de rugosidades en el paladar y desarrollo relativo de las crestas craneanas. Otras manifestaciones de la variabilidad intraespecífica en los ejemplares analizados son la presencia o ausencia de cúspulas sobre los cíngulos anterobasales de los molares inferiores, la eventual ausencia de M4, el grado de desarrollo del foramen infraorbitario y el número, tamaño y disposición de los forámenes mentonianos.

La variabilidad encontrada en los distintos ejemplares de Arctodictis munizi aquí estudiados concuerda con el amplio rango de diferenciación intraespecífica observada 
en los grandes mamíferos carnívoros placentarios actuales. Las diferencias en estos últimos están fundamentalmente relacionadas con el tamaño corporal; tal es el caso de los osos (Kurtén, 1967), felinos (Kurtén, 1973, 1985) y hienas (Kurtén, 1973). Parte de esta variabilidad, sobre todo en las especies con amplio rango de distribución, resulta ser de tipo clinal; no obstante, no deben descartarse los componentes sexuales e individuales, además de los temporales (Kurtén, 1967, 1973). Sobre este último componente, debe tenerse en cuenta que los ejemplares aquí revisados no proceden todos del mismo nivel estratigráfico.

\section{RELACIONES FILOGENÉTICAS}

Los objetivos del presente análisis son: (a) reconocer los caracteres diagnósticos del género Arctodictis, (b) determinar las especies que lo componen y (c) contrastar la hipótesis de que los ejemplares UCMP 39250 y MLP 85-VII-3-1 son asignables a especies de este género. A tal efecto, fueron considerados los siguientes taxones y ejemplares de Sparassodonta: Lycopsis longirostrus, Prothylacynus patagonicus, Borhyaena tuberata, Arctodictis munizi, A. sinclairi, y los ejemplares UCMP 39250 y MLP 85-VII-3-1. Un detalle de los materiales analizados se ofrece en la Tabla 1.

Fueron considerados 24 caracteres, de los cuales 19 son de tipo binario y 5 son multiestado. La lista de caracteres está organizada de la siguiente manera: caracteres 1 a 15 , rasgos craneanos; 16 a 23, dentarios; 24, postcráneo. Lycopsis longirostrus fue considerado como grupo externo dado que este taxón constituye, en el análisis cladístico presentado por Muizon (1999), un clado externo al grupo aquí analizado.

Sobre la matriz de caracteres elaborada (Tabla 5) se aplicó el algoritmo de simplicidad de NONA (Goloboff, 1993), bajo la interfaz de WINCLADA (Nixon, 1999).

\section{Análisis de caracteres}

1. Apertura del borde posterior del canal infraorbitario. Anterior al M1 (0); sobre el M1 (1).

2. Foramen palatino posterior (posterolateral). Cerrado, limitado por un puente óseo (0); abierto (1). Sobre este carácter,
Marshall (1977c: 416) señala que "phylogenetic inference based on this highly variable character is of low reliability" no obstante, será considerado hasta que no sea demostrado su carácter de no informativo para los boriénidos.

3. Expansión del paladar por detrás del último molar. Ausente (0); presente (1). Tomado de Rougier et al. (1998; carácter 94).

4. Número de fosas intermolares del paladar. Con una sola concavidad entre el M3 y el M4 (0); con dos concavidades: entre M2 y M3 y entre M3 y M4 (1); ambos estados (2).

5. Diámetro transverso de la cavidad glenoidea. Menos del doble del diámetro anteroposterior (0); más del doble del diámetro anteroposterior (1). Tomado de Muizon (1999; carácter 19; véase también Rougier et al., 1998, carácter 71). Muizon (1999) considera que el estado 1 está presente en Prothylacynus; sin embargo, en los ejemplares estudiados por nosotros, se observó el estado 0.

6. Ubicación del foramen postglenoideo. Ubicado en el sector medio del proceso postglenoideo (0); o más próximo al plano sagital del cráneo (1).

7. Apertura del foramen oval. Delimitado sólo por el aliesfenoides (0); entre aliesfenoides y el petroso (1). Carácter modificado de Marshall (1977c; carácter 5) y Muizon (1999, carácter 7). Algunos autores (Marshall, 1977c; Muizon, 1999) sugieren que en Borhyaena el foramen lacerado medio confluye con el foramen oval verdadero, por lo cual debería denominarse foramen pseudoval. Asimismo, Muizon (1999) propone un estado distinto para aquellos Sparassodonta que presentan foramen pseudoval. En el presente trabajo, sin embargo, preferimos codificar los estados en relación con el hueso que lo circunda, independientemente de si existe o no confluencia de otros canales en él.

8. Foramen del canal transverso. Presente (0); ausente (1). Tomado de Marshall (1977c; carácter 4), Rougier et al. (1998; carácter 104) y Muizon (1999; carácter 8).

9. Apertura del foramen carotídeo. El foramen carotídeo abre a una altura media del basiesfenoides (0); abre a la altura de la sutura entre el basioccipital y el basiesfenoides (1). Modificado de Rougier et al. (1998, carácter 5) y Sánchez Villagra et al. (2000, carácter 6).

10. Concavidad del aliesfenoides contribuyendo al sinus hipotimpánico. Presente (0); ausente (1).

11. Quilla del basioccipital. Ausente (0); presente (1).

12. Premaxilar acuñado entre el maxilar y el nasal. En forma poco notoria (0), en forma marcada (1).

13. Sínfisis mandibular. Hemimandíbulas articuladas entre sí (0); fuertemente anquilosadas, con fusión sólo en el sector dor-

\begin{tabular}{lcccccccccccccccccccccccccc}
\hline taxones/caracteres & 1 & 2 & 3 & 4 & 5 & 6 & 7 & 8 & 9 & 10 & 11 & 12 & 13 & 14 & 15 & 16 & 17 & 18 & 19 & 20 & 21 & 22 & 23 & 24 \\
\hline Lycopsis longirostrus & 0 & 0 & $?$ & 0 & 0 & 0 & 0 & 0 & 0 & $?$ & 0 & $?$ & 0 & 1 & 0 & $?$ & 0 & 0 & 0 & 0 & 0 & 1 & 0 & 0 \\
Prothylacynus patagonicus & 0 & 0 & 0 & 1 & 0 & 1 & 0 & 0 & 0 & $?$ & 1 & 1 & 2 & 0 & 1 & 0 & 1 & 0 & 0 & 0 & 0 & 0 & 0 & 0 \\
Borhyaena tuberata & 0 & 0 & 0 & 2 & 1 & 1 & 1 & 1 & 1 & 0 & $?$ & 0 & 0 & 1 & 0 & 1 & 1 & 1 & 1 & 0 & 0 & 2 & 0 & 1 \\
UCMP 39250 & $?$ & $?$ & $?$ & 0 & 0 & 1 & $?$ & $?$ & $?$ & $?$ & $?$ & $?$ & 0 & $?$ & $?$ & $?$ & 1 & $?$ & 0 & 0 & 0 & $?$ & $?$ & 0 \\
MLP 85-VII-3-1 & 1 & 1 & 1 & 0 & 1 & 1 & 1 & 1 & 1 & 1 & 0 & 1 & 1 & 1 & 0 & 1 & 2 & 1 & 1 & 1 & 1 & 2 & 0 & 1 \\
Arctodictis munizi & 1 & 0 & 1 & 0 & 1 & 1 & 1 & 1 & 1 & 1 & 0 & 0 & 2 & 1 & 0 & 1 & 2 & 1 & 1 & 1 & 2 & 2 & 1 & $?$ \\
Arctodictis sinclairi & $?$ & $?$ & $?$ & $?$ & $?$ & $?$ & $?$ & $?$ & $?$ & $?$ & $?$ & $?$ & 1 & $?$ & 0 & $?$ & 2 & 1 & 1 & 1 & 1 & $?$ & $?$ & $?$
\end{tabular}

Tabla 5. Matriz de datos. Data matrix. 
sal (1); totalmente fusionadas entre sí (2). Carácter modificado de Muizon (1999; carácter 35); este autor considera sólo dos estados: sínfisis articuladas o fusionadas. Marshall (1978) incluyó un estado intermedio para Arctodictis sinclairi, postura que seguimos aquí.

14. Ángulo entre el borde ventral de la rama horizontal y la cresta infracondilar. Con ángulo de casi $90^{\circ}(0)$; describiendo una suave curva (1). Carácter tomado de Rougier et al. (1998, carácter 69).

15. Forámenes mentonianos subiguales en tamaño. Ausente (0); presente (1).

16. Número de incisivos. $4 / 3$ (0); $3 / 3$ (1). Modificado de Marshall (1977c, carácter 1), Rougier et al. (1998, carácter 8) y Muizon (1999, carácter 37).

17. Implantación del P/p 1. P1 implantado en forma levemente oblicua con respecto a la hilera dentaria (0); oblicuo (1); implantado en forma transversal a la hilera dentaria (2). Carácter modificado de Muizon (1999; carácter 24). Este autor considera conjuntamente la longitud y ancho del paladar y del rostro y la implantación del P/p 1. Dado que muchos de los ejemplares comparados representan restos fragmentarios, se prefirió considerar sólo la implantación del P/p 1.

18. Tamaño de los premolares 2 y 3. $\mathrm{P} / \mathrm{p} 2-\mathrm{P} / \mathrm{p} 3$ subiguales entre sí (0); P/p3 mucho más grande que el P/p2 (1). Modificado de Rougier et al. (1998, carácter 3).

19. Desarrollo del talónido en los molares inferiores. Con talónido desarrollado (0); con talónido vestigial (1).

20. Lóbulo descendente de la corona sobre la raíz posterior de los m1-3. Ausente (0); presente (1).

21. Raíces "globosas". Ausencia (0); raíces globosas sólo en el p2 (1); en todos los premolares (2). El estado 2 se encuentra exclusivamente en Arctodictis munizi; adicionalmente, en el ejemplar MLP 11-85 de esta última especie, el carácter se manifiesta también en los primeros molares.
22. Grado de desarrollo de la plataforma estilar. Plataforma estilar amplia, con desarrollo de cúspides estilares (0); reducción de plataforma y cúspides estilares (1); ausente o vestigial (2).

23. Desarrollo de la cúspula posterior del P3. Poco desarrollada (0); bien desarrollada (1).

24. Foramen entepicondilar del húmero. Presente (0); ausente (1).

Comentario sobre los "complejos funcionales" en la delimitación de caracteres de la morfología dentaria.

Muizon (1999, carácter 42) reúne varios caracteres en uno solo: adaptación dentaria al carnivorismo, considerando un complejo funcional relacionado con la dieta carnívora, y que incluye los siguientes rasgos: oclusión predominante de tipo prevallid-postvallum; reducción o pérdida del metacónido, aumento de la superficie cortante formada por el paracónido y protocónido; reducción del talónido; reducción del protocono; tendencia a la fusión del paracono a la base del metacono, con reducción del paracono; aumento de la postmetacrista; tendencia a la reducción o pérdida de la plataforma estilar. Sin embargo, hemos considerado necesaria la evaluación de ciertos caracteres por separado; tal es el caso de los caracteres 19 (desarrollo del talónido) y 22 (grado de desarrollo de la plataforma estilar) dado que la variación de dichos caracteres se manifiestan de manera independiente entre los taxones aquí analizados.

\section{Resultados}

Como resultado del análisis filogenético se obtuvo el cladograma de la figura 8 . Se desactivaron las autapomor-

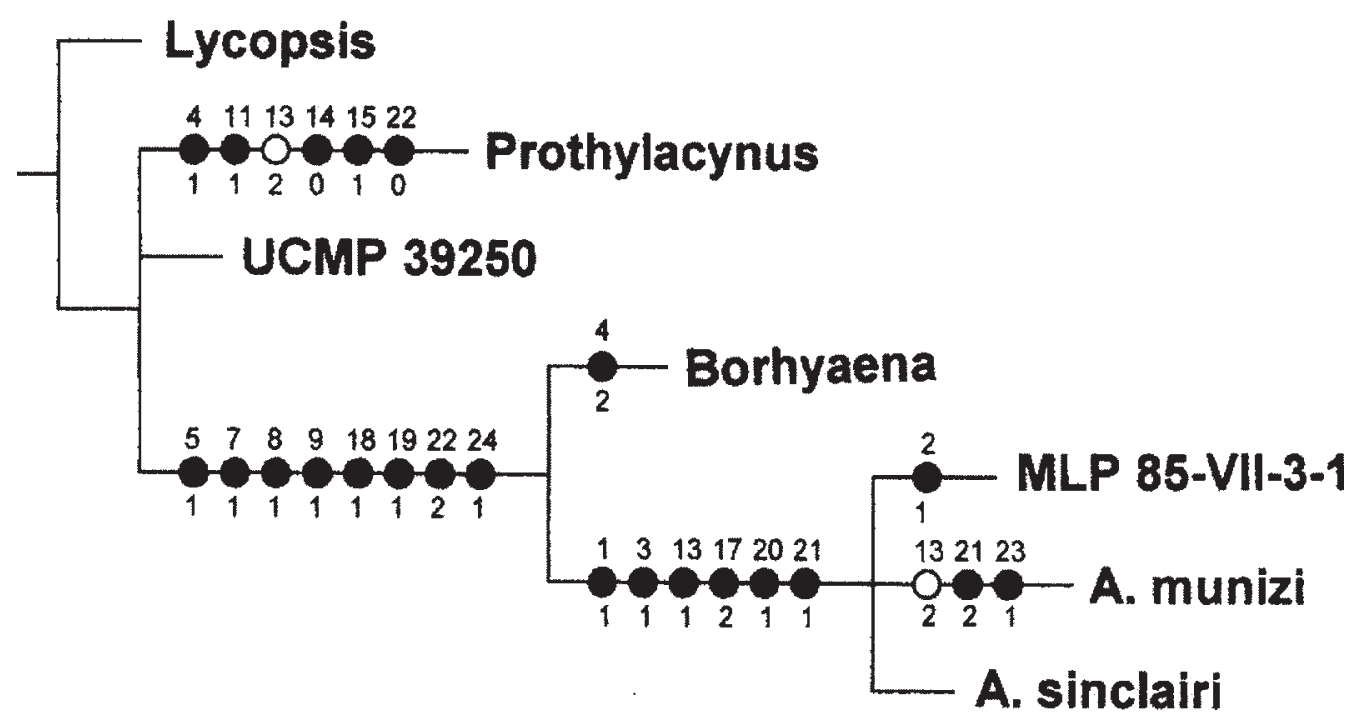

Figura 8. Cladograma resultante del análisis filogenético realizado en este trabajo. Los círculos en negro representan sinapomorfías o autapomorfías; los círculos blancos representan homoplasias. Los números por encima y por debajo de cada círculo representan el número de carácter y el estado del mismo respectivamente (véase el análisis de caracteres).

Cladogram resultant of the phylogenetic analysis made in this paper. The black dots mean synapomorphies or autapomorphies; the white dots mean homoplasies. The numbers above and below of each dot mean the number of the character and the character state respectively (see the analysis of the characters). 
fías (caracteres $2,11,15$ y 23) y se consideraron como aditivos los caracteres 17 y 22. Los parámetros del árbol obtenido son los siguientes: longitud: 27 , ci: 87 , ri: 82 . Se aprecia que el género Arctodictis constituye un grupo natural compuesto por dos especies: A. munizi y $A$. sinclairi (en la que se incluye tentativamente el espécimen MLP 85-VII-3-1, véase más abajo). Los caracteres compartidos entre ambas especies (véase Fig. 8) son los que en el presente trabajo se consideran diagnósticos para el género Arctodictis. De este modo, se propone una nueva diagnosis la cual difiere parcialmente de la previamente establecida por Marshall (1978) ya que no se contemplan los caracteres plesiomórficos.

El ejemplar asignado tentativamente por Marshall (1978) a Arctodictis (UCMP 39250) constituye una forma más afín a Prothylacynus que a cualquiera de las especies del género Arctodictis o que a cualquier otro Borhyaeninae.

Para el universo taxonómico aquí considerado, Borhyaena representa el grupo hermano de Arctodictis. Sin embargo, debe tenerse en cuenta que varios géneros de Borhyaeninae no fueron incorporados en la matriz, por lo que con la inclusión de otros taxones estas relaciones podrían modificarse. En el presente análisis excluímos a Pharsophorus, considerado por Marshall (1978: 69) como forma ancestral de Arctodictis, debido al carácter fragmentario de los restos disponibles, los cuales incorporaban numerosos interrogantes a la matriz y por consiguiente una muy baja resolución en el análisis.

\section{DISCUSIÓN Y CONCLUSIONES}

Actodictis munizi constituye la especie más grande del género y uno de los más grandes metaterios sudamericanos hasta ahora conocidos. Los siguientes caracteres mandibulares y dentarios sugieren hábitos osífragos para Arctodictis munizi: (1) la notable robustez del cuerpo mandibular, rasgo apreciable no sólo en el ancho sino en la altura de la rama mandibular desde el canino hasta el último molar; (2) la fusión de la sínfisis mandibular, (3) la notoria robustez de las raíces de la dentición postcanina y (4) la presencia de numerosas microfracturas en el esmalte dentario. Por otra parte, los caracteres diagnósticos de esta especie son: presencia de una sínfisis mandibular fusionada (carácter 13), de raíces globosas en todos los premolares (carácter 21) y de una cúspula posterior en el P3 (carácter 23) bien desarrollada.

Arctodictis sinclairi difiere de A. munizi por su menor tamaño, el cuerpo del dentario menos robusto, la sínfisis mandibular fusionada sólo en el sector más dorsal y por el carácter globoso de las raíces sólo en el p2.

Los resultados del análisis filogenético sugieren la pertenencia del ejemplar MLP 85-VII-3-1 al género Arctodictis. Debido a que adicionalmente presenta aque- llos rasgos propios de A. sinclairi (mencionados más arriba) se asigna tentativamente este ejemplar a dicha especie. Existen algunas diferencias entre el ejemplar MLP 85VII-3-1 y el ejemplar tipo (tamaño algo menor que el FMNH P13526, ausencia de una cúspula posterobasal en el p3 y contorno más redondeado del borde anterolingual de este mismo premolar). Sin embargo, teniendo en cuenta el rango de variabilidad observada en los ejemplares asignados a $A$. munizi, interpretamos este conjunto de diferencias como parte de la variabilidad intraespecífica de la especie A. sinclairi.

Marshall (1976a: 418; 1977b: 633) mencionó la existencia de un boriénido de gran talla (el ejemplar UCMP 39250) procedente de niveles de la Edad Laventense (Mioceno Medio) de La Venta, Colombia. Posteriormente (Marshall, 1978: 58) lo consideró como cf. Arctodictis sp. El material consiste en fragmentos craneanos y postcraneanos asociados. Según este autor, consiste en un individuo juvenil, dado que se ha preservado la cripta del p3 no erupcionado. Un nuevo examen del ejemplar en cuestión sugiere que no corresponde a un juvenil por los siguientes argumentos: el M4 está completamente erupcionado (en otros boriénidos, como en Prothylacynus patagonicus MACN A 5922, al menos el m4, el P3 y el M4 erupcionan simultáneamente); el único molar preservado (m1 o $\mathrm{m} 2$ izquierdo) muestra un desgaste moderado; las epífisis de los elementos postcraneanos están completamente fusionadas; y finalmente, la identificación mencionada por Marshall (1978: 58) de una "cripta" para el p3 en el fragmento mandibular derecho es por lo menos confusa, ya que sólo se ha preservado la parte anterior del alvéolo de este diente.

Los argumentos dados por Marshall para justificar la asignación de este ejemplar a cf. Arctodictis sp. son: tamaño grande, estructura masiva de los caninos (cuyas raíces muestran surcos longitudinales), e implantación oblicua del p1 "...and to a less extent the p2" (Marshall, 1978: 58). Sin embargo, ninguno de estos rasgos es exclusivo de Arctodictis sino que aparecen con frecuencia en otros Borhyaenidae. Por otra parte, de la observación del ejemplar UCMP 39250 se destaca que el carácter oblicuo del p1 es apenas moderado, mientras que el p2 está implantado casi paralelo al eje dentario, una condición claramente distinta de aquella observable en Arctodictis. La sínfisis mandibular no fusionada fue interpretada por Marshall (1978) como consecuencia del carácter juvenil del ejemplar; sin embargo, otros esparasodontes muestran este rasgo independientemente de su edad. Otros rasgos confrontables, como la gracilidad de la rama mandibular, el carácter más angosto y largo de la cavidad glenoidea, el fuerte desarrollo del talónido (en lugar de una cúspula posterior) en el único molar inferior preservado, y la ausencia de un lóbulo basal descendente sobre la raíz posterior de los molares, excluyen la pertenencia de este ejemplar al género Arctodictis y a los Borhyaeninae en general. 
En conclusión: (1) el ejemplar UCMP 39250 no corresponde a un individuo juvenil, (2) no existen rasgos, más allá de su gran tamaño, que permitan asignarlo al género Arctodictis o a algún otro Borhyaeninae conocido. Goin (1997) sugirió la posible pertenencia de este ejemplar a la especie Dukecynus magnus (Borhyaenidae, "Prothylacyninae"). La presencia de rasgos tales como: raíces de los dientes no fuertemente infladas; p1 implantado oblicuamente, aunque de forma poco marcada y talónidos bien desarrollados, presentes tanto en el UCMP 39250 como en la especie tipo del género Dukecynus (Goin, 1997) sugiere una mayor afinidad a este género que a otro Borhyaenidae hasta ahora conocido.

\section{AGRADECIMIENTOS}

A los Dr. José Bonaparte, del Museo Argentino de Ciencias Naturales "Bernardino Rivadavia" (Argentina) y Dr. Guillermo Rougier de la University of Louisville (Kentucky), por los valiosos comentarios al manuscrito original; a los Dr. S. Vizcaíno, del Museo de La Plata (Argentina), Dr. Richard Fariña, del Museo Nacional de Historia Natural de Montevideo (Uruguay), Dr. María L. Martínez Chacón y Dr. Sixto Fernández López por las correcciones al manuscrito; al Dr. William Clemens y colaboradores del Departamento de Paleontología de la Universidad de California (Berkeley) por brindar el acceso a las colecciones; y a los Sres. Jorge González, Agustín Viñas y Maximiliano Lezcano por los dibujos que ilustran este trabajo.

\section{BIBLIOGRAFÍA}

Ameghino, F. 1887. Enumeración sistemática de las especies de mamíferos fósiles coleccionados por Carlos Ameghino en los terrenos eocenos de la Patagonia austral y depositados en el Museo de La Plata. Boletín del Museo de La Plata, 1, 1-26.

Ameghino, F. 1891. Observaciones críticas sobre los mamíferos eocenos de Patagonia austral. Revista Argentina de Historia Natural, 1, 328-380.

Ameghino, F. 1894. Enumération synoptique des espèces de mammifères fósiles des formations éocenes de Patagonia. Boletín de la Academia Nacional de Ciencias, Córdoba, 13, 259-445.

Ameghino, F. 1906. Les formations sédimentaires du Crétacé supérieur et du Tertiaire de Patagonia avec un paralélle entre leurs faunes et celles de l'ancient continent. Anales del Museo Nacional de Historia Natural de Buenos Aires, 15, 1-568.

Archer, M. 1976. The dasyurid dentition and its relationships to that of didelphids, thylacynids, borhyaenids (Marsupicarnivora) and peramelids (Peramelina: Marsupialia). Australian Journal of Zoology (supplementary series), 39, 1-34.

Argot, C, 2001. Functional-adaptative anatomy of the forelimb in the Didelphidae, and the paleobiology of the Pa- leocene marsupials Mayulestes ferox and Pucadelphys andinus. Journal of Morphology, 247, 51-79.

Cabrera, A. 1927. Datos para el conocimiento de los dasyuroideos fósiles argentinos. Revista del Museo de La Plata, 30, 271-315.

Chelotti, L. A. 1992. Rasgos tectónicos del área del medio y bajo río Coyle, Cuenca Austral. Revista de la Asociación Geológica Argentina, 47, 129-134.

Evans, H. E. y deLahunta, A. 1972. Disección del Perro de Miller. Ed. Interamericana. México, primera edición en español, $282 \mathrm{pp}$.

Goin, F. J. 1997. New clues for understanding Neogene marsupial radiations. In: A History of the Neotropical Fauna. Vertebrate paleobiology of the Miocene in Colombia (Eds. R. F. Kay, R. H. Madden, R. L. Cifelli and J. Flynn). Smithsonian Institution Press, Washington, 185-204.

Goloboff, P. A. 1993. NONA Computer programs and documentation. New York. Free download at www. cladistics.org

Hatcher, J. B. 1903. Narrative of the expedition. Reports Princeton University Expeditions to Patagonia (18961899). In: Bone hunters in Patagonia: narrative of the expedition. Ox Bow Press, Woodbridge, 1985, 209 pp.

Hershkovitz, P. 1982. The staggered marsupial lower third incisor (I3). Géobios (mémoire spécial), 6, 191-200.

Hiiemae, K. and Jenkins, F. A. 1969. The anatomy and internal architecture of the muscles of mastication in Didelphis marsupialis. Postilla, 140, 1-49.

Huxley, T. H. 1880. On the application of the laws of evolution to the arrangement of the Vertebrata, and more particularly of the Mammalia. Proceedings of the Zoological Society of London, 43, 649-662.

Kurtén, B. 1967. Pleistocene bear of North America 2. Genus Arctodus. Short-faced bears. Acta Zoologica Fennica, 117, 1-60.

Kurtén, B. 1973. Geographic variation in size in the puma (Felis concolor). Commentationes Biologicae, 63, 1-8.

Kurtén, B. 1985. The Pleistocene lion of Beringia. Annales Zoologici Fennici, 22, 117-121.

Marshall, L. G. 1976a. New Didelphine Marsupials from the La Venta fauna (Miocene) of Colombia, South America. Journal of Paleontology, 50, 402-418.

Marshall, L. G. 1976b. Fossil localities for Santacrucian (Early Miocene) mammals, Santa Cruz Province, Southern Patagonia, Argentina. Journal of Paleontology, 50, 1129-1142.

Marshall, L. G. 1977a. Evolution of the carnivorous adaptative zone in South America. In: Major patterns in vertebrate evolution (Eds. M. K. Hecht, P. C. Goody and B. M. Hecht), Plenum press, New York, 709-722.

Marshall, L. G. 1977b. A new species of Lycopsis (Borhyaenidae: Marsupialia) from the La Venta fauna (Late Miocene) of Colombia, South America. Journal of $\mathrm{Pa}$ leontology, 51, 633-642.

Marshall, L. G. 1977c. Cladistic analysis of borhyaenid, dasyuroid, didelphoid and thylacynid (Marsupialia: Mammalia) affinity. Systematic Zoology, 26, 410-425.

Marshall, L. G. 1978. Evolution of the Borhyaenidae, extinct 
South American predaceous marsupials. University of California Publications in Geological Sciences, 117, 1-89.

Marshall, L. G. 1979. Review of the Prothylacyninae, an extinct subfamily of South American "dog-like" marsupials. Fieldiana Geology (new series), 3, 1-50.

Marshall, L. G. 1981. Review of the Hathlyacyninae, an extinct subfamily of South American "dog-like" marsupials. Fieldiana Geology, 7, 1-120.

Marshall, L. G. 1985. Geochronology and land-mammal biochronology of the Transamerican faunal interchange. In: The Great American Biotic Interchange (Eds. F. Stehli and S. D. Webb). Plenum Press, New York, 49-85.

Marshall, L. G. and Cifelli, R. L. 1990. Analysis of changing diversity patterns in Cenozoic Land Mammal Age Faunas, South America. Paleovertebrata, 19, 169-210.

Marshall, L. G. and Kielan-Jaworowska, Z. 1992. Relationships of the dog-like marsupials, deltatheroidans and early tribosphebic mammals. Lethaia, 25, 361-374.

Marshall, L. G., Hoffstetter, R. and Pascual, R. 1983. Geochronology of the continental mammal-bearing Tertiary of South America. Palaeovertebrata (mémoire extraordinaire), 1-93.

Marshall, L. G., Cifelli, R. L., Drake, R. E. and Curtis, G. H. 1986. Geochronology of type Santacrucian (Middle Tertiary) land mammal age, Patagonia, Argentina. Journal of Geology, 94, 449-457.

Marshall, L. G., Case, J. A. and Woodburne, M. O. 1990. Phylogenetic relationships of the families of marsupials. Current Mammalogy, 2, 433-502.

Mercerat, A. 1891. Caracteres diagnósticos de algunas especies de Creodonta conservadas en el Museo de La Plata. Revista del Museo de La Plata, 2, 51-56.

Muizon, C. de 1994. A new carnivorous marsupial from the early Palaeocene of Bolivia and the problem of marsupial monophyly. Nature, 370, 208-211.

Muizon, C. de 1998. Mayulestes ferox, a borhyaenoid (Metatheria, Mammalia) from the early Palaeocene of Bolivia. Phylogenetic and palaeobiologic implications. Geodiversitas, 20, 19-142.

Muizon, C. de 1999. Marsupial skulls from the Deseadan (Late Oligocene) of Bolivia and Phylogenetic analysis of the Borhyaenoidea (Marsupialia, Mammalia). Geobios, 32, 483-509.

Nixon, K. C. 1999. Winclada (Beta) ver. 0.9.9. Publicada por el autor. Ithaca, NY.

Osgood, W. H. 1921. A monographic study of the American marsupials, Caenolestes. Field Museum of Natural History (zoological series), 14, 1-162.

Pardiñas, U. J. F. 1991. Primer registro de primates y otros vertebrados para la Formación Collón Curá (Mioceno Medio) del Neuquén, Argentina. Ameghiniana, 28, 197-199.
Petter, G and Hoffstetter, R. 1983. Les marsupiaux du Déséadien (Oligocène Inférieur) de Salla (Bolivie). Annales de Paléontologie (vert.-invert.), 69, 175-234.

Rougier, G. W., Wible, J. R. and Novacek, M. J. 1998. Implications of Deltatheridium specimens for early marsupials history. Nature, 396, 459-463.

Sánchez Villagra, M. R., Kay, R. F. and Anaya Daza, F. 2000. Cranial anatomy and palaeobiology of the Miocene marsupial Hondalagus altiplanensis and a phylogeny of argyrolagids. Palaeontology, 43, 287-301.

Scillato Yané, G. J. 1986. Los Xenarthra fósiles de Argentina (Mammalia, Edentata). In: Simposio "Evolución de los vertebrados cenozoicos", $4^{\circ}$ Congreso Argentino de Paleontología y Bioestratigrafía, 2, 151-155.

Scott, W. B. 1905. Mammalia of the Santa Cruz Beds. Part. 1 Edentata. Reports Princeton University Expedition to Patagonia (1896-1899), 5, 1-364.

Simpson, G. G. 1980. Splendid Isolation. The curious history of South American Mammals. Yale University Press, New Haven, 266 pp.

Sinclair, W. J. 1906. Mammalia of the Santa Cruz Beds. Marsupialia. Reports of the Princeton University Expedition to Patagonia, 4, 333-460.

Sinclair, W. J. 1909. Mammalia of the Santa Cruz Beds: Typotheria. Reports Princeton University Expedition to Patagonia (1896-1899), 4, 1-110.

Tauber, A. A. 1991. Homunculus patagonicus Ameghino, 1891 (Primates, Ceboidea), del Mioceno Temprano de la costa atlántica austral, provincia de Santa Cruz, Argentina. Academia Nacional de Ciencias, Córdoba, Miscelánea, 82,1-32.

Tauber, A. A. 1994. Estratigrafía y vertebrados fósiles de la Formación Santa Cruz (Mioceno Inferior) en la costa atlántica de Santa Cruz, República Argentina. Tesis Doctoral, Facultad de Ciencias Exactas, Físicas y Naturales, Universidad Nacional de Córdoba, República Argentina, 422 pp. (inédita).

Tauber, A. A. 1996. Los representantes del género Protyptherium (Mammalia, Notoungulata, Interatheriidae) del Mioceno Temprano del sudeste de la provincia de Santa Cruz, República Argentina. Academia Nacional de Ciencias, Córdoba, Miscelánea, 95, 1-29.

Tauber, A. A. 1997a. Bioestratigrafía de la Formación Santa Cruz (Mioceno Inferior) en el extremo sudeste de la Patagonia. Ameghiniana, 34, 413-426.

Tauber, A. A. 1997b. Paleoecología de la Formación Santa Cruz (Mioceno Inferior) en el extremo sudeste de la Patagonia. Ameghiniana, 34, 517-529.

Tauber, A. A. 1999. Los vertebrados de la Formación Santa Cruz (Mioceno Inferior-Medio) en el extremo sureste de la Patagonia y su significado paleoecológico. Revista Española de Paleontología, 14, 173-182. 